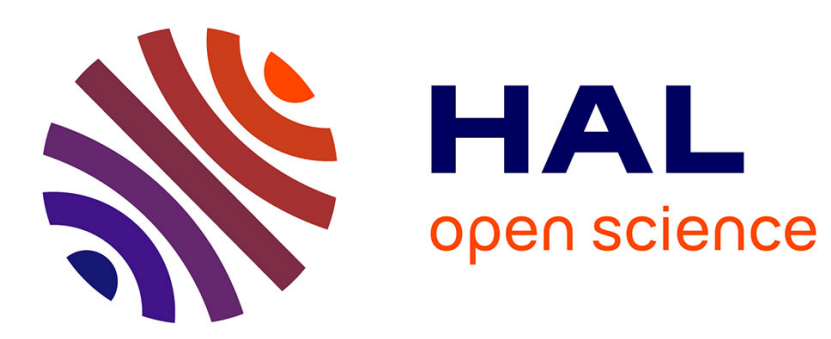

\title{
Instabilities of convection patterns in a shear-thinning fluid between plates of finite conductivity
}

\author{
Thomas Varé, Chérif Nouar, Christel Metivier
}

\section{To cite this version:}

Thomas Varé, Chérif Nouar, Christel Metivier. Instabilities of convection patterns in a shear-thinning fluid between plates of finite conductivity. Physical Review E , 2017, 96 (4), pp.43109 - 43109. 10.1103/PhysRevE.96.043109 . hal-02442198v2

\section{HAL Id: hal-02442198 \\ https://hal.univ-lorraine.fr/hal-02442198v2}

Submitted on 28 Nov 2017 (v2), last revised 16 Jan 2020 (v3)

HAL is a multi-disciplinary open access archive for the deposit and dissemination of scientific research documents, whether they are published or not. The documents may come from teaching and research institutions in France or abroad, or from public or private research centers.
L'archive ouverte pluridisciplinaire HAL, est destinée au dépôt et à la diffusion de documents scientifiques de niveau recherche, publiés ou non, émanant des établissements d'enseignement et de recherche français ou étrangers, des laboratoires publics ou privés. 


\title{
Instabilities of convection patterns in a shear-thinning fluid between plates of finite conductivity
}

\author{
Thomas Varé, Chérif Nouar, ${ }^{*}$ and Christel Métivier \\ LEMTA UMR 7563 CNRS-Université de Lorraine, ENSEM, 2 Avenue de la Forêt de Haye, TSA 60604-54516 \\ Vandoeuvre lès Nancy cedex, France \\ (Received 13 July 2017; published 26 October 2017)
}

\begin{abstract}
Rayleigh-Bénard convection in a horizontal layer of a non-Newtonian fluid between slabs of arbitrary thickness and finite thermal conductivity is considered. The first part of the paper deals with the primary bifurcation and the relative stability of convective patterns at threshold. Weakly nonlinear analysis combined with Stuart-Landau equation is used. The competition between squares and rolls, as a function of the shear-thinning degree of the fluid, the slabs' thickness, and the ratio of the thermal conductivity of the slabs to that of the fluid is investigated. Computations of heat transfer coefficients are in agreement with the maximum heat transfer principle. The second part of the paper concerns the stability of the convective patterns toward spatial perturbations and the determination of the band width of the stable wave number in the neighborhood of the critical Rayleigh number. The approach used is based on the Ginzburg-Landau equations. The study of rolls stability shows that: (i) for low shear-thinning effects, the band of stable wave numbers is bounded by zigzag instability and cross-roll instability. Furthermore, the marginal cross-roll stability boundary enlarges with increasing shear-thinning properties; (ii) for high shear-thinning effects, Eckhaus instability becomes more dangerous than cross-roll instability. For square patterns, the wave number selection is always restricted by zigzag instability and by "rectangular Eckhaus" instability. In addition, the width of the stable wave number decreases with increasing shear-thinning effects. Numerical simulations of the planform evolution are also presented to illustrate the different instabilities considered in the paper.
\end{abstract}

DOI: 10.1103/PhysRevE.96.043109

\section{INTRODUCTION}

Studies on patterns formation and their stability in Rayleigh-Bénard convection for Newtonian fluids have been considered in several papers. A review can be found in books of Getling [1] and Koschmieder [2] and more recently in Bodenschatz et al. [3] where the most significant progress in the field is identified. Comparatively to the Newtonian case, only a limited number of studies were devoted to non-Newtonian fluids and still fewer to nonlinear developments. Yet, non-Newtonian fluids intervene in a very broad range of industrial processes such as polymer and foodstuffs processing and in complex physical phenomena such as the convective movements in the Earth's mantle. Here, we focus on the shear-thinning behavior, i.e., nonlinear decrease of the effective viscosity with the shear rate, which is the most common property of non-Newtonian fluids. In recent articles [4-6], the nature and the stability of patterns that emerge in Rayleigh-Bénard convection for shear-thinning fluids have been studied using a weakly nonlinear analysis. Boussinesq approximations have been adopted and the slabs have been considered as perfectly conducting. Using Carreau model to describe the shear-thinning behavior of the fluid, it has been shown in Ref. [6] that: (i) rolls are the only stable convective patterns at threshold and (ii) there is a critical value of the shear-thinning degree $\alpha$ defined by Eq. (12) above which the bifurcation becomes subcritical.

Most analyses consider ideal situations where the bounding horizontal surfaces are perfect conductors of heat. However, in many laboratory experiments and in engineering and geophysical problems, the slabs have a finite conductivity and they are no better conductor than the fluid itself. In this case, the temperature disturbances do not vanish on the boundaries. The

*Corresponding author: cherif.nouar@univ-lorraine.fr thermal boundary conditions that have to be satisfied are the continuity of temperature and heat flux. According to Cerisier et al. [7], the temperature fluctuation occurring in the liquid close to a nearly insulating slab distorts the temperature distribution. This temperature distortion can lead to an instability of the fluid layer. As a consequence, at threshold, the temperature gradient is small and the fluid organizes in a pattern with a small wave number. Furthermore, theoretical and experimental studies show that squares may be the convection patterns at the onset instead of rolls. Experimental evidence of square patterns was reported by Legal, Pocheau, and Croquette [8] and Legal and Croquette [9]. The competition between roll and square patterns for a Newtonian fluid has been examined in weakly supercritical Rayleigh-Bénard convection by Busse and Riahi [10], Proctor [11], and Jenkins and Proctor [12]. The results are presented in terms of the Prandtl number Pr and the ratio $\chi$ of the thermal conductivities of slabs and fluid. It has been shown, for instance, that for $\operatorname{Pr} \geqslant 10$ and for slabs with thickness equal to the width of the fluid layer, that the convective pattern at threshold is in the form of squares when $\chi<\chi_{c}=1$. These studies were extended recently to shear-thinning fluids by Bouteraa and Nouar [13]. It has been found that the critical value $\chi_{c}$, below which squares are stable, decreases with increasing shear-thinning effects. Recently, experiments were done by Kebiche [14], using carboxymethylcellulose (CMC) solutions as shear-thinning fluid. In the Rayleigh-Bénard setup, the slabs are made of polycarbonate with a ratio of thermal conductivities $\chi \approx 0.25$. PIV measurements done in one vertical section do not allow the determination of convection pattern type.

These studies are valid only in the immediate vicinity of the threshold with perfectly periodic pattern. However, as the Rayleigh number $\mathrm{Ra}$ is increased above the onset $\mathrm{Ra}_{\mathrm{c}}$, the growth-rate of the perturbation is positive for any wave number within a band $\sqrt{\epsilon}$ with $\epsilon=\left(\mathrm{Ra}-\mathrm{Ra}_{\mathrm{c}}\right) / \mathrm{Ra}_{\mathrm{c}}$, 
around the critical wave number $k_{c}$. A wave packet centered on $k_{c}$ can be constructed with the unstable modes. The corresponding convective pattern is modulated spatially on a scale of $O(1 / \sqrt{\epsilon})$. The stability analysis of these convective patterns with respect to long wavelength perturbations is of great interest. It allows, in particular, the determination of the range of stable wave numbers. Typical instability mechanisms are Eckhaus (E), zigzag (ZZ), and cross-roll (CR) instabilities [15]. Eckhaus instability is a phase instability that acts on the roll phase to change the wavelength, compressing or dilating the pattern. Zigzag is also a phase instability that arises from perturbations parallel to the roll axes: it creates undulations along the roll axes when the wavelength is too large. Cross-roll instability is an amplitude instability that consists of a set of rolls growing perpendicularly to the original pattern. For a Newtonian fluid with perfectly heat conductive slabs, the instability mechanisms that tend to limit the stability region of rolls depend on the Prandtl number and on the boundary conditions, rigid or stress-free boundary conditions, as shown by Busse [15] and Bolton and Busse [16]. At large Prandtl number, say $\operatorname{Pr}>10$, with no-slip conditions the region of stable convection rolls is bounded by the zigzag instability and the cross-roll instability, which is followed by the bimodal convection when $\mathrm{Ra}$ is increased. At low Prandtl number, say $\operatorname{Pr}<1$, Eckhaus instability becomes more dangerous than cross-roll instability and the domain of stable zigzag enlarges as Pr decreases. Furthermore, other specific secondary instabilities like "skewed-varicose instability" and oscillatory instability [17] participate in bounding the stability domain of rolls. Generally, non-Newtonian fluids are highly viscous and so the corresponding Prandtl number is large. Therefore, only universal secondary instabilities-Eckhaus, zigzag, and cross-roll-are considered in this paper.

Square patterns are also subject to long wavelength instabilities. In the case of poorly conducting slabs and for Newtonian fluids, Hoyle [18] has shown that the range of stable wave numbers is restricted by zigzag instability and by Eckhaus rectangular instability. According to Hoyle [18], this latter instability has a three-dimensional character since the system responds differently in each of the two horizontal directions. It behaves like one of two rolls that constitute square pattern and grows locally at the expense of the other. This is why Holmedal et al. [19] called this instability "Long wavelength cross-roll instability." This study was extended by Holmedal [19] to the general case of slabs with different finite conductivities and thicknesses of the slabs.

The objective of this paper is to investigate the influence of shear-thinning effects on the stability of the convective patterns and the width of the stable band of wave numbers in Rayleigh-Bénard convection with slabs of finite conductivity and arbitrary thickness. The rheological law introduces additional nonlinearity and coupling between flow variables. This additional nonlinearity will induce stronger interactions between the two sets of rolls that constitute square patterns than in the Newtonian case. Therefore, shear-thinning effects will modify not only the range of stable wave number but also the more restrictive instability mechanism. A weakly nonlinear analysis based on the amplitude equations formalism [18,20] is adopted as a first approach to examine nonlinear effects arising from the rheology.

To our knowledge, the present study is the first one that considers the influence of the rheology on secondary instabilities. The structure of the paper is as follows. In Sec. II the problem is formulated. Linear stability analysis is briefly considered in Sec. III. The nature of the primary bifurcation and pattern selection are discussed in Sec. IV. It is observed that shear-thinning effects favor formation of rolls. The stability of the convective patterns with respect to inhomogeneous spatial perturbations is investigated in Sec. V, using the amplitude equations formalism. Influence of shear-thinning effects is highlighted. In Sec. VI, time evolution of the convective pattern is illustrated from the numerical simulation of amplitude equations. The paper ends with a conclusion where the relevant results are summarized.

\section{PROBLEM FORMULATION}

We consider a shear-thinning fluid layer of infinite horizontal extent that is heated from below and cooled from above. We assume the rigid slabs that enclose the fluid have arbitrary conductivities and thicknesses. The thermal conductivity and diffusivity are noted $\hat{K}$ and $\hat{\kappa}$ for the fluid and $\hat{K}_{p}$ and $\hat{\kappa}_{p}$ for the slabs. We define $\chi$ as the ratio of $\hat{K}_{p}$ and $\hat{K}$ and we assume as in Refs. $[11,21]$ that $\chi=\frac{\hat{K_{p}}}{\hat{K}}=\frac{\hat{\kappa_{p}}}{\hat{\kappa}}$. This assumption is reasonable for several couples (fluid, slab) where the ratio of the thermal capacities of the fluid and the slabs $r=\frac{\left(\hat{\rho} \hat{C}_{p}\right)_{\text {fluid }}}{\left(\hat{\rho} \hat{C}_{p}\right)_{\text {slabs }}}$ remains of order 1: for instance, $r$ (water,copper) $=1.22$ and $r($ glycerin, glass $)=1.67$.

Dimensional quantities are denoted with the symbol hat $\left(^{\wedge}\right)$. In the following, we note $\hat{d}$ the depth of the fluid layer, $\Delta \hat{T}=$ $\hat{T}_{1}-\hat{T}_{2}$, the temperature difference between the outer surfaces of the upper and lower slabs. Because of the thermal expansion, the temperature difference between the two plates induces a vertical density stratification. Heavy cold fluid is above a warm light fluid. For small $\Delta \hat{T}$, the fluid remains motionless and the heat is transferred by conduction with a linear temperature profile across the fluid layer. In the fluid, $-\hat{d} / 2<\hat{z}<\hat{d} / 2$, the hydrostatic solution and the temperature profile are

$$
\begin{aligned}
\frac{d \hat{P}}{d \hat{z}} & =-\hat{\rho}_{0} \hat{g}\left[1-\hat{\beta}\left(\hat{T}-\hat{T}_{0}\right)\right] \\
\text { and } \quad \hat{T}_{\text {cond }} & =\hat{T}_{0}-\frac{\Delta \hat{T}}{1+2 \Lambda / \chi} \frac{\hat{z}}{\hat{d}},
\end{aligned}
$$

where $\hat{g}$ is the acceleration due to gravity and $\Lambda$ the dimensionless thickness of slabs. The $z$ axis is directed upwards with the origin located at the middle of the fluid layer. The reference temperature $\hat{T}_{0}$ is the temperature at the middle of the fluid layer, $\hat{\rho}_{0}$ the fluid density at $\hat{T}_{0}$, and $\hat{\mu}_{0}$ is the zero-shear rate viscosity at $\hat{T}_{0}$. The temperature difference between the top and the bottom of the fluid layer is $\Delta \hat{T}_{f}=\Delta \hat{T}[1+2 \Lambda / \chi]$. The temperature profiles in top and bottom plates are

$$
\begin{aligned}
\hat{T}_{\text {cond }} & =\hat{T}_{0}+\frac{\Delta \hat{T}}{2 \Lambda+\chi}\left[\frac{1}{2}-\frac{1}{2} \chi-\frac{\hat{z}}{\hat{d}}\right] ; \\
\frac{\hat{d}}{2} & \leqslant \hat{z} \leqslant\left(\frac{1}{2}+\Lambda\right) \hat{d}
\end{aligned}
$$


and

$$
\begin{aligned}
\hat{T}_{\text {cond }}= & \hat{T}_{0}+\frac{\Delta \hat{T}}{2 \Lambda+\chi}\left[\frac{1}{2} \chi-\frac{1}{2}-\frac{\hat{z}}{\hat{d}}\right] ; \\
& -\left(\Lambda+\frac{1}{2}\right) \hat{d} \leqslant \hat{z} \leqslant-\frac{\hat{d}}{2} .
\end{aligned}
$$

When the top and bottom plates are poor thermal conductors, a large part of $\Delta \hat{T}$ occurs across the plates and remains only a small part $\Delta \hat{T}_{f}$ of $\Delta \hat{T}$, acting as a driving force for the convection. When $\Delta \hat{T}_{f}$ exceeds a critical value, the convection sets in and convective patterns emerge. The stability of the hydrostatic solution is considered by introducing temperature and pressure perturbations as well as a fluid motion. The fluid is incompressible and Boussinesq approximations are adopted. We use $\hat{d}, \hat{\mu}_{0}, \frac{\hat{d}^{2}}{\hat{\kappa}}, \frac{\hat{\kappa}}{\hat{d}}, \frac{\hat{\rho}_{\hat{\kappa}} \hat{\kappa}^{2}}{\hat{d}^{2}}$, and $\frac{\Delta \hat{T}}{\mathrm{Ra}}$ as characteristic scales of length, viscosity, time, velocity, pressure, and temperature, respectively. Using these scales, the perturbation equations read

$$
\begin{aligned}
\nabla \cdot \mathbf{v} & =0, \\
\frac{1}{\operatorname{Pr}}\left(\frac{\partial \mathbf{v}}{\partial t}+(\mathbf{v} \cdot \nabla) \mathbf{v}\right) & =-\nabla p+\theta \mathbf{e}_{\mathbf{z}}+\nabla \cdot \boldsymbol{\tau}, \\
\frac{\partial \theta}{\partial t}+(\mathbf{v} \cdot \nabla) \theta & =\operatorname{Ra} w+\Delta \theta, \\
\frac{\partial \theta_{p}}{\partial t} & =\chi \Delta \theta_{p},
\end{aligned}
$$

where $\mathbf{v}=u \mathbf{e}_{x}+v \mathbf{e}_{y}+w \mathbf{e}_{z}, p$ are, respectively, the velocity and pressure perturbations, $\theta$ and $\theta_{p}$ are temperature perturbations in the fluid and the slabs, respectively, and $\boldsymbol{\tau}$ is the deviatoric of the stress tensor. The Prandtl (Pr) and Rayleigh (Ra) numbers are defined by

$$
\operatorname{Pr}=\frac{\hat{\mu_{0}}}{\hat{\rho}_{0} \hat{\kappa}}, \quad \operatorname{Ra}=\frac{\hat{\rho_{0}} \hat{g} \hat{\beta} \Delta \hat{T} \hat{d}^{3}}{\hat{\kappa} \hat{\mu}_{0}} .
$$

We consider a purely viscous shear-thinning fluid,

$$
\boldsymbol{\tau}=\mu(\Gamma) \dot{\boldsymbol{\gamma}},
$$

where $\Gamma$ is the second invariant of the strain-rate tensor:

$$
\Gamma=\frac{1}{2} \dot{\gamma}_{i j} \dot{\gamma}_{i j} ; \quad \dot{\gamma}_{i j}=\frac{\partial v_{i}}{\partial x_{j}}+\frac{\partial v_{j}}{\partial x_{i}},
$$

where $v_{i}$ are the components of the velocity and $x_{i}$ are the spatial coordinates.

Concerning the nonlinear rheological law $\mu(\Gamma)$, we have used a Carreau model [22]. In dimensional form, it is given by

$$
\frac{\hat{\mu}-\hat{\mu}_{\infty}}{\hat{\mu}_{0}-\hat{\mu}_{\infty}}=\left(1+\hat{\lambda}^{2} \hat{\Gamma}\right)^{\frac{n_{c}-1}{2}},
$$

where $\hat{\mu}_{\infty}$ is the infinite-shear viscosity, $\hat{\mu}_{0}$ the zero-shear viscosity, $\hat{\lambda}$ a characteristic time of the fluid, $n_{c}$ the shearthinning index. For several polymer solutions, $\hat{\mu}_{\infty} \ll \hat{\mu}_{0}$ [23]. Hence, neglecting $\hat{\mu}_{\infty}$ with respect to $\hat{\mu}_{0}$, we have in dimensionless form

$$
\mu=\left(1+\lambda^{2} \Gamma\right)^{\frac{n_{c}-1}{2}} .
$$

A Taylor series expansion of $\mu$ around the base state (where the fluid is at rest) allows us to define the degree of shear-thinning of the fluid as

$$
\alpha=\left|\frac{d \mu}{d \Gamma}\right|_{\Gamma=0}=\frac{1-n_{c}}{2} \lambda^{2} .
$$

No-slip and nonpenetration boundary conditions as well as continuity of temperature and heat flux at the interface slabsfluid read

$$
\begin{gathered}
\mathbf{v}\left(z= \pm \frac{1}{2}\right)=\mathbf{0}, \\
\theta\left(z= \pm \frac{1}{2}\right)=\theta_{p}\left(z= \pm \frac{1}{2}\right), \\
\frac{\partial \theta}{\partial z}\left(z= \pm \frac{1}{2}\right)=\chi \frac{\partial \theta_{p}}{\partial z}\left(z= \pm \frac{1}{2}\right) .
\end{gathered}
$$

Temperatures of the outer surfaces of the upper and lower slabs are fixed, thus,

$$
\theta_{p}\left(z=\frac{1}{2}+\Lambda\right)=\theta_{p}\left(z=-\frac{1}{2}-\Lambda\right)=0 .
$$

In the momentum equations, the pressure field can be eliminated using the curl of Eq. (5). We then take the curl of Eq. (5) one more time. Using the continuity equation, and projecting onto $\boldsymbol{e}_{z}$, we get the following evolution equations for the vertical vorticity $\zeta$ and the vertical velocity $w$ :

$$
\begin{gathered}
\frac{1}{\operatorname{Pr}}\left[\frac{\partial \zeta}{\partial t}+\boldsymbol{e}_{z} \cdot \nabla \times[(\boldsymbol{v} \cdot \nabla) \boldsymbol{v}]\right] \\
=\Delta \zeta+\boldsymbol{e}_{z} \cdot \nabla \times[\nabla \cdot(\mu-1) \dot{\boldsymbol{\gamma}}], \\
\frac{1}{\operatorname{Pr}}\left[\frac{\partial \boldsymbol{\nabla}^{2} w}{\partial t}-\boldsymbol{e}_{z} \cdot[\boldsymbol{\nabla} \times \boldsymbol{\nabla} \times[(\boldsymbol{v} \cdot \boldsymbol{\nabla}) \boldsymbol{v}]]\right] \\
=\Delta^{2} w+\nabla_{H}^{2} \theta-[\nabla \times \nabla \times[\nabla \cdot(\mu-1) \dot{\boldsymbol{\gamma}}]] \cdot \boldsymbol{e}_{z}, \\
\frac{\partial \theta}{\partial t}+(\boldsymbol{v} \cdot \boldsymbol{\nabla}) \theta=\operatorname{Ra} w+\nabla^{2} \theta, \\
\frac{\partial \theta_{p}}{\partial t}=\chi \nabla^{2} \theta_{p},
\end{gathered}
$$

where

$$
\zeta=\frac{\partial v}{\partial x}-\frac{\partial u}{\partial y} \quad \text { and } \quad \nabla_{H}^{2}=\frac{\partial^{2}}{\partial x^{2}}+\frac{\partial^{2}}{\partial y^{2}} .
$$

From the continuity equation and the vertical vorticity definition, one can deduce the horizontal velocity components $(u, v)$ :

$$
\nabla_{H}^{2} u=-\frac{\partial^{2} w}{\partial x \partial z}-\frac{\partial \zeta}{\partial y} ; \quad \nabla_{H}^{2} v=-\frac{\partial^{2} w}{\partial y \partial z}+\frac{\partial \zeta}{\partial x} .
$$

The boundary conditions for $w$ are

$$
w=D w=0 \quad \text { at } \quad z= \pm 1 / 2 .
$$

For the temperature, the boundary conditions are

$$
\begin{gathered}
\theta_{p}=0 \quad \text { at } \quad z= \pm(1 / 2+\Lambda), \\
\theta=\theta_{p} \quad \text { at } \quad z= \pm 1 / 2, \\
D \theta=\chi D \theta_{p} \quad \text { at } \quad z= \pm 1 / 2 .
\end{gathered}
$$


Five dimensionless parameters appear in the governing equations: the Rayleigh number Ra, the Prandtl number Pr, the thermal conductivities ratio $\chi$, the dimensionless thickness of the slab $\Lambda$, and the shear-thinning degree $\alpha$. In the present study, $\operatorname{Pr}=10$. Actually, our results do not vary significantly with $\operatorname{Pr}$ when $\operatorname{Pr} \geqslant 10$.

\section{LINEAR STABILITY ANALYSIS}

\section{A. Critical conditions}

For infinitesimal perturbations, the Boussinesq Eqs. (17)-(20) are linearized, and one obtains

$$
\begin{gathered}
\frac{1}{\operatorname{Pr}} \frac{\partial \zeta}{\partial t}=\Delta \zeta, \\
\frac{1}{\operatorname{Pr} \frac{\partial \Delta w}{\partial t}}=\Delta^{2} w+\operatorname{Ra} \Delta_{H} \theta \\
\frac{\partial \theta}{\partial t}=w+\Delta \theta \\
\frac{\partial \theta_{p}}{\partial t}=\chi \nabla^{2} \theta_{p} .
\end{gathered}
$$

At the linear level, the rheology of the fluid does not play any role. Furthermore, the vertical vorticity decouples and obeys a diffusion Eq. (26) and thus can be neglected in the linear theory. Assuming a spatial periodicity in the horizontal plane, we seek a normal mode solution under the form

$$
\left[\begin{array}{c}
w(x, y, z, t) \\
\theta(x, y, z, t) \\
\theta_{p}(x, y, z, t)
\end{array}\right]=\left[\begin{array}{c}
F_{11}(z) \\
G_{11}(z) \\
G_{p 11}(z)
\end{array}\right] \exp (i \boldsymbol{k} \cdot \boldsymbol{r}+s t),
$$

where $\boldsymbol{r}=(x, y)$ is the vector position in the horizontal plane, and $\boldsymbol{k}$ is the wave vector. Substituting Eq. (30) into Eqs. (27)(29) leads to the differential equations

$$
\begin{gathered}
s \operatorname{Pr}^{-1}\left(D^{2}-k^{2}\right) F_{11}=-k^{2} \operatorname{Ra} G_{11}+\left(D^{2}-k^{2}\right)^{2} F_{11}, \\
s G_{11}=F_{11}+\left(D^{2}-k^{2}\right) G_{11}, \\
s G_{p 11}=\chi\left(D^{2}-k^{2}\right) G_{p 11},
\end{gathered}
$$

with $k=|k|$. The boundary conditions are

$$
\begin{gathered}
F_{11}=D F_{11}=0 \quad \text { at } \quad z=0,1, \\
G_{p 11}=0 \quad \text { at } \quad z=-\Lambda, 1+\Lambda, \\
G_{11}=G_{p 11} \quad \text { at } \quad z=0,1, \\
D G_{11}=\chi D G_{p 11} \quad \text { at } z=0,1 .
\end{gathered}
$$

The eigenvalue problem Eqs. (31)-(33) with the boundary conditions Eqs. (34)-(37) is solved using a Chebyshev collocation method. The marginal stability curve $\operatorname{Ra}(k)$ is determined by the condition $s=0$. The minimum of the marginal stability curve gives the critical Rayleigh number $\mathrm{Ra}_{\mathrm{c}}$ and critical wave number $k_{c}$. We recover the results of Ref. [13] for $\Lambda=1$ and we extend them to other thicknesses $\Lambda$ on Fig. 1. We observe that $k_{c}$ and $\mathrm{Ra}_{\mathrm{c}}$ decrease with decreasing the ratio $\chi$ of conductivities. Actually, $k_{c}$ and $\mathrm{Ra}_{\mathrm{c}}$

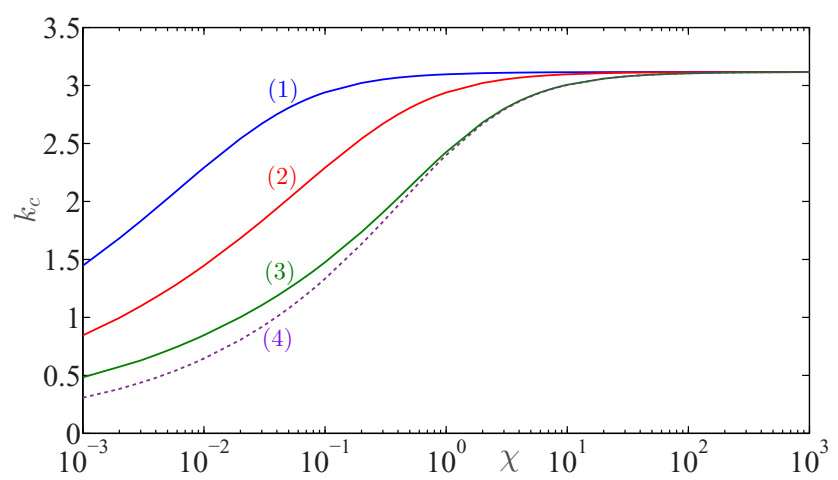

(a)

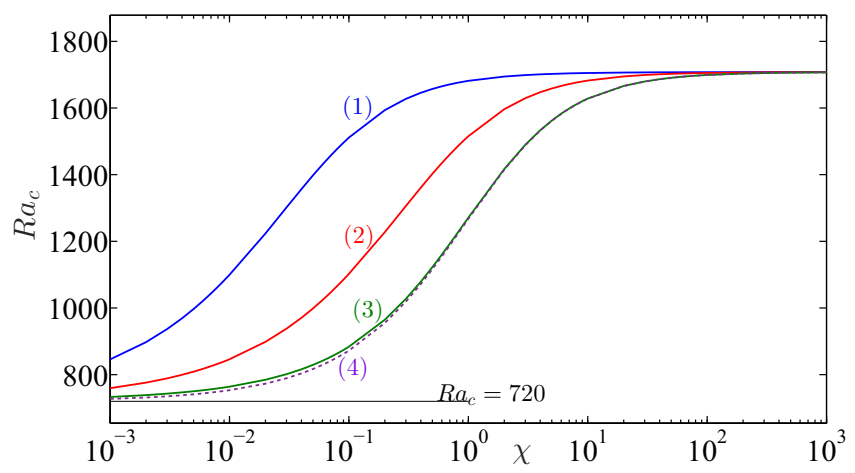

(b)

FIG. 1. Evolution of the critical wave number $k_{c}$ (a) and the critical Rayleigh number $\mathrm{Ra}_{\mathrm{c}}$ (b) as a function of ratio of the thermal conductivities $\chi$ for different values of the slab thickness $\Lambda$ : (1) $\Lambda=0.01$, (2) $\Lambda=0.1$, (3) $\Lambda=1$, (4) $\Lambda=10$.

vary from, respectively, 3.11 and 1708 to 0 and 720 [11,24]. An explanation of this evolution can be found in Ref. [25] in the limit of perfectly insulating slabs. From a physical point of view, such configuration means that the temperature field is fixed in the solid (or evolves on a very long time scale compared to that of the fluid). As a consequence, the temperature gradient, and therefore the energy flux, is fixed in the solid. Hence, the temperature fluctuations at the interface do not propagate inside the solid and primary bifurcation needs less energy to occur, which explains the decrease of $\mathrm{Ra}_{\mathrm{c}}$ with decreasing $\chi$.

Remark. Linear stability analysis gives the critical Rayleigh number $\mathrm{Ra}_{\mathrm{c}}$ for instability onset and determines the modulus $k_{c}$ of the critical wave-vector $\boldsymbol{k}$ of the unstable modes. The direction of $\boldsymbol{k}$ is arbitrary. This orientation degeneracy is related to the isotropy of the horizontal plane [26]. There is also a pattern degeneracy that results from the linear theory itself; indeed, any superposition of normal modes,

$$
\begin{aligned}
& {\left[w(\boldsymbol{r}, z), \theta(\boldsymbol{r}, z), \theta_{p}(\boldsymbol{r}, z)\right]} \\
& \quad=\sum_{\ell} c_{\ell} \exp \left(i \boldsymbol{k}_{\ell} \cdot \boldsymbol{r}\right)\left[F_{11}(z), G_{11}(z), G_{11 p}(z)\right],
\end{aligned}
$$

with $\left|\boldsymbol{k}_{\ell}\right|=k_{c}$ and where the $c_{\ell}$ 's are constant coefficients, is also a solution of the linear problem with a zero growth rate at criticality. 


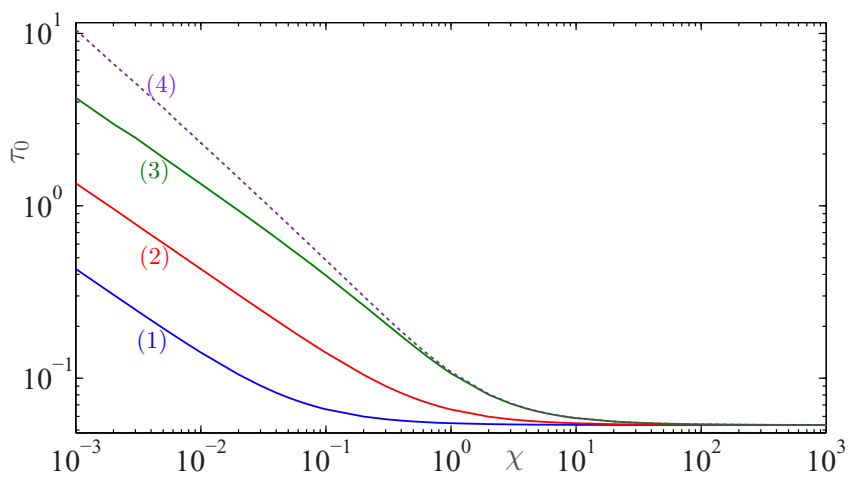

FIG. 2. Evolution of $\tau_{0}$ versus $\chi$ for $\operatorname{Pr}=10$ and different values of $\Lambda$. (1) $\Lambda=0.01$, (2) $\Lambda=0.1$, (3) $\Lambda=1$, (4) $\Lambda=10$.

\section{B. Characteristic time of the instability}

Near the onset of convection, the growth rate $s$ of the perturbation may be approximated using Taylor expansion,

$$
s=\frac{\epsilon}{\tau_{0}}+O\left(\epsilon^{2}\right) \quad \text { with } \quad \epsilon=\frac{\mathrm{Ra}-\mathrm{Ra}_{\mathrm{c}}}{\mathrm{Ra}_{\mathrm{c}}},
$$

where $\tau_{0}$ is the characteristic time for the instability to grow. It is given by $\tau_{0}=\operatorname{Ra}_{\mathrm{c}}\left(\frac{\partial s}{\partial \mathrm{Ra}}\right)_{k_{c}, \mathrm{Ra}_{\mathrm{c}}}$. It is obtained from the curve, temporal amplification rate $s$ versus Rayleigh number $\mathrm{Ra}$, at the critical conditions. Its evolution is represented in Fig. 2. The increase of $\tau_{0}$ with decreasing $\chi$ is related to the increase of the thermal diffusion time in the slab as $\hat{k}_{p}$ diminishes. For $\chi>10$, we recover the value corresponding to a perfect heat conductor, $\tau_{0}=0.053$. Note that $\tau_{0}$ does not depend on the rheological parameters.

\section{PATTERN SELECTION AT THE ONSET OF CONVECTION}

The selection of the convective pattern is determined by the nonlinearities of the problem, i.e., nonlinear inertial and nonlinear viscous terms. A weakly nonlinear analysis based on amplitude expansion method similar to that considered in Refs. [6,27-30] is used as a first approach to investigate nonlinear effects on the competition between convective patterns. Actually, the pattern that emerges near the onset of convection are either rolls or squares. Further calculations show that hexagons are unstable $[6,12]$.

\section{A. Principles of the amplitude expansion method: Case of square}

For a square pattern, the fundamental solution in the linear regime is $\left(A e^{i \boldsymbol{k}_{1} \cdot \boldsymbol{r}}+B e^{i \boldsymbol{k}_{2} \cdot \boldsymbol{r}}\right) \psi_{11}$, with $\boldsymbol{k}_{2}$ orthogonal to $\boldsymbol{k}_{1}$ (the two vectors $\boldsymbol{k}_{1}$ and $\boldsymbol{k}_{2}$ have the same modulus), $A$ and $B$ are the complex amplitudes of the perturbation along the two wave vectors and $\psi_{11}(z)$ stands for $F_{11}(z), G_{11}(z)$, or $G_{p 11}(z)$. The interaction of the fundamental solution with itself, through the quadratic nonlinear inertial terms produces the first harmonic $\left(A^{2} e^{2 i \boldsymbol{k}_{1} \cdot \boldsymbol{r}}+B^{2} e^{2 i \boldsymbol{k}_{2} \cdot \boldsymbol{r}}\right) \psi_{22}$ and a coupling between modes $\boldsymbol{k}_{1}$ and $\boldsymbol{k}_{2}, A B e^{i\left(\boldsymbol{k}_{1}+\boldsymbol{k}_{2}\right) \cdot \boldsymbol{r}} \psi_{A B}$. The interaction of the fundamental with its complex conjugate leads to an other coupling between modes $\boldsymbol{k}_{1}$ and $\boldsymbol{k}_{2}, A B^{*} e^{i\left(\boldsymbol{k}_{1}-\boldsymbol{k}_{2}\right) \cdot \boldsymbol{r}} \psi_{A B^{*}}$, where (.) denotes the complex conjugate and a correction of the base state, $\left(\left|A^{2}\right|+\left|B^{2}\right|\right) \psi_{02}$. The feedback at the cubic order on the fundamental solution through nonlinear inertial and viscous terms is $\left(\left|A^{2}\right|+\left|B^{2}\right|\right)\left(A e^{i \boldsymbol{k}_{1} \cdot \boldsymbol{r}}+B e^{i \boldsymbol{k}_{2} \cdot \boldsymbol{r}}\right) \psi_{13}$. From this cascade of nonlinear interactions, the nonlinear solution can be written as

$$
\begin{aligned}
\psi(\boldsymbol{r}, z, t)= & \left(A(t) e^{i \boldsymbol{k}_{1} \cdot \boldsymbol{r}}+B(t) e^{i \boldsymbol{k}_{2} \cdot \boldsymbol{r}}\right) \psi_{11}(z)+\text { c.c. }+\left(A^{2}(t) e^{2 i \boldsymbol{k}_{1} \cdot \boldsymbol{r}}+B^{2}(t) e^{2 i \boldsymbol{k}_{2} \cdot \boldsymbol{r}}\right) \psi_{22}(z)+A(t) B(t) e^{i\left(\boldsymbol{k}_{1}+\boldsymbol{k}_{2}\right) \cdot \boldsymbol{r}} \psi_{A B}(z)+\text { c.c. } \\
& +\left(\left|A^{2}(t)\right|+\left|B^{2}(t)\right|\right) \psi_{02}(z)+A(t) B^{*}(t) e^{i\left(\boldsymbol{k}_{1}-\boldsymbol{k}_{2}\right) \cdot \boldsymbol{r}} \psi_{A B^{*}}(z)+\text { c.c. }+\left(\left|A^{2}(t)\right|+\left|B^{2}(t)\right|\right)\left(A(t) e^{i \boldsymbol{k}_{1} \cdot \boldsymbol{r}}\right. \\
& \left.+B(t) e^{i \boldsymbol{k}_{2} \cdot \boldsymbol{r}}\right) \psi_{13}(z)+\text { c.c. }+\ldots
\end{aligned}
$$

In Eq. (40), $\psi(\boldsymbol{r}, z, t)$ stands for the vertical velocity perturbation, $w$, or the temperature perturbation $\theta$ or $\theta_{p}$. For the vertical velocity perturbation, $\psi_{i j}$ is denoted $F_{i j}$, and for the temperature perturbation, $\psi_{i j}$ is denoted $G_{i j}$.

In the square lattice, time evolution of the amplitude perturbations is governed by Stuart-Landau amplitude equations,

$$
\begin{aligned}
& \frac{d A}{d t}=\frac{\epsilon}{\tau_{0}} A-\left(g_{1}|A|^{2}+\beta|B|^{2}\right) A, \\
& \frac{d B}{d t}=\frac{\epsilon}{\tau_{0}} B-\left(g_{1}|B|^{2}+\beta|A|^{2}\right) B,
\end{aligned}
$$

where $g_{1}$ and $\beta$ are, respectively, self-saturation and crossedsaturation coefficients. The form of the amplitude Eqs. (41) and (42) is completely determined by the rules of invariance via symmetry by rotation of an angle $\pi / 2$ and by translation [31,32]. Substituting Eqs. (40)-(42) into Eqs. (18)-(20) yields after some algebra to a set of differential equations for each mode that are solved sequentially. To avoid secular terms at the cubic order, compatibility conditions have to be enforced using the Fredholm alternative. The latter states that the resonating forcing terms have to be orthogonal to the kernel of the adjoint of the linear operator. This allows the determination of landau saturation coefficients $g_{1}$ and $\beta$.

\section{B. Nature of the primary bifurcation}

As shown in Ref. [6], the self-saturation $g_{1}$ and crossed saturation $\beta$ coefficients can be written as the sum of Newtonian ( $N$ superscript) and non-Newtonian contributions ( $n N$ superscript):

$$
\begin{gathered}
g_{1}=g_{1}^{N}-\alpha g_{1}^{n N}, \\
\beta=\beta^{N}-\alpha \beta^{n N} .
\end{gathered}
$$

It is therefore possible to define a critical value $\alpha_{c}$ of the shear-thinning degree above which the bifurcation becomes subcritical. 


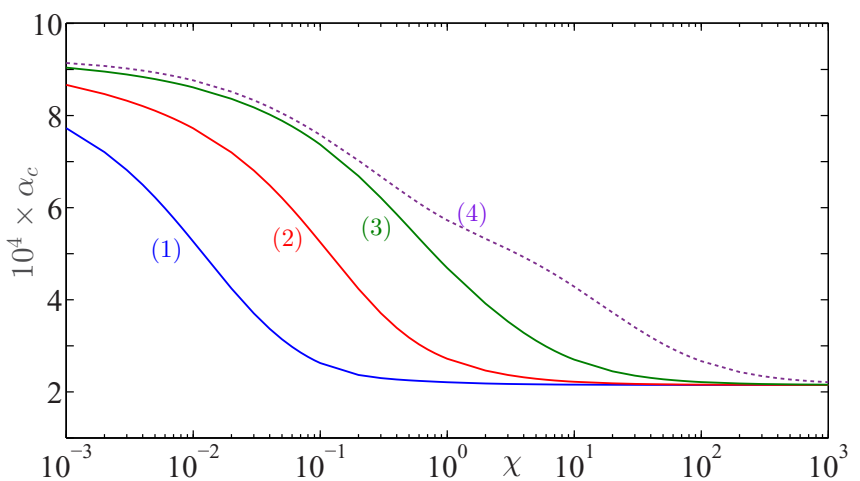

(a) Rolls

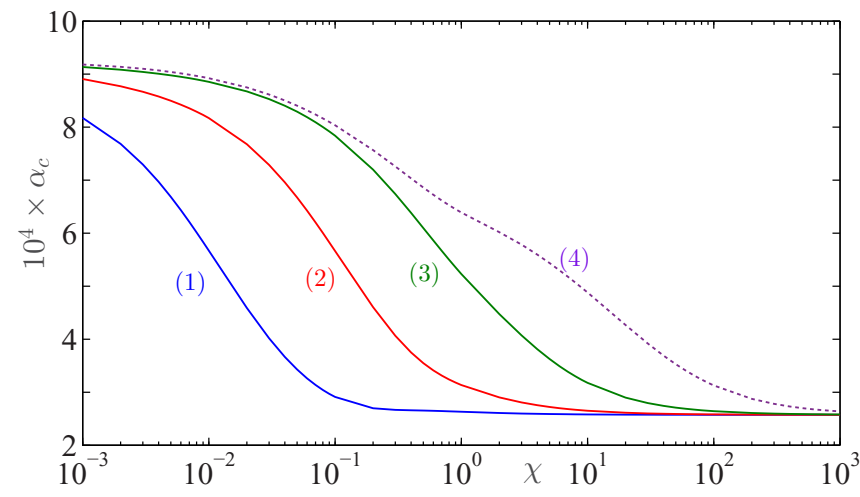

(b) Squares

FIG. 3. Critical degree of shear-thinning, above which the primary bifurcation becomes subcritical, versus $\chi$ for rolls (a) and squares (b). (1) $\Lambda=0.01$, (2) $\Lambda=0.1$, (3) $\Lambda=1$, (4) $\Lambda=10$.

In the case of rolls, $\beta=0$ and

$$
\alpha_{c}=\frac{g_{1}^{N}}{g_{1}^{n N}} .
$$

In the case of squares, $\beta \neq 0$ and

$$
\alpha_{c}=\frac{g_{1}^{N}+\beta^{N}}{g_{1}^{n N}+\beta^{n N}} .
$$

Variations of $\alpha_{c}$ with $\chi$ for different $\Lambda$ are depicted in Figs. 3(a) and 3(b) for rolls and squares respectively. For large $\chi$, the asymptotic limit of $\alpha$ is $\alpha_{c}=2.15 \times 10^{-4}$ in agreement with Refs. $[4,6]$. With decreasing $\hat{K}_{p}$ (decreasing $\chi)$, the intensity of convection decreases, therefore, it is not surprising that stronger shear-thinning effects are needed to obtain a subcritical bifurcation.

\section{Convective patterns at threshold}

A linear stability analysis of stationary roll and square solutions of Eqs. (41) and (42) allows to show that squares are stable when $\beta<g_{1}$, i.e., when the coupling between the two orthogonal modes that describe the square pattern is weak enough. By contrast, when $\beta>g_{1}$, the coupling is too strong, the squares lose their stability and rolls are the stable nonlinear state. A similar phenomenological description can be found in Ref. [26]. Figure 4 shows that with increasing $\alpha, \frac{\beta}{g_{1}}$ increases, and thus the interaction between the two orthogonal modes $\boldsymbol{k}_{1}$ and $\boldsymbol{k}_{2}$ becomes stronger. A possible

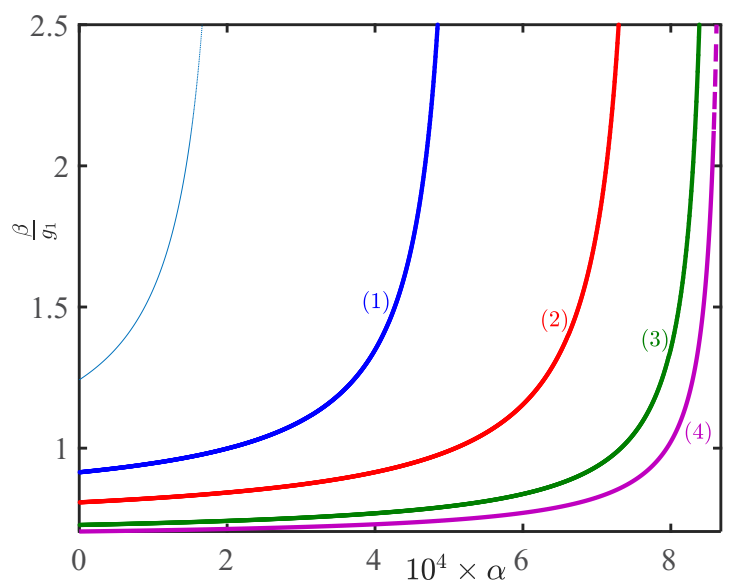

FIG. 4. The ratio $\frac{\beta}{g_{1}}$ as a function of $\alpha$ for $\chi=10^{-2}$ and different values of $\Lambda$ : (1) $\Lambda=0.01$, (2) $\Lambda=0.1$, (3) $\Lambda=1$, (4) $\Lambda=10$. The thin curve corresponds to the case of prefect heat conductor plates. It is represented as a reference curve.

interpretation may be related to the reduction of viscosity with increasing shear-thinning effects, which leads to an increase of the convection intensity. Nonlinearities and coupling between modes become stronger which favor roll patterns.

Using shear-thinning decomposition of $g_{1}$ and $\beta$ [Eqs. (43) and (44)], it is found that rolls are stable when $\alpha>\alpha_{S-R}$, with

$$
\alpha_{S-R}=\frac{\beta^{N}-g_{1}^{N}}{\beta^{n N}-g_{1}^{n N}} .
$$

Stability domains of squares and rolls are represented in the plane $(\chi, \alpha)$ for different $\Lambda$ in Fig. 5. The curves represent the boundaries between squares and rolls: below the boundary, squares are stable, and above the boundary, rolls are the stable convective patterns. For the limit of Newtonian fluids, i.e., $\alpha=0$, we recover the results of the literature [12]: for $\operatorname{Pr} \geqslant 10$ and $\Lambda=1$, rolls are stable patterns provided that $\chi>\chi_{c}=1$. We notice that the domain of stability of squares

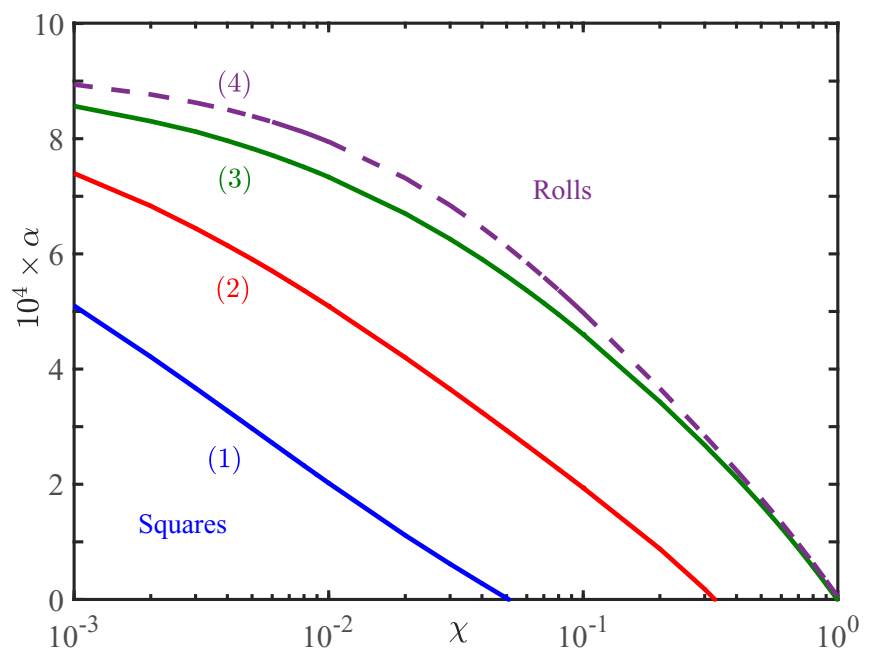

FIG. 5. Stability domains of rolls and squares as a function of $\chi$ and $\alpha$ for different values of $\Lambda$ : (1) $\Lambda=0.01$, (2) $\Lambda=0.1$, (3) $\Lambda=1$, (4) $\Lambda=10$. 


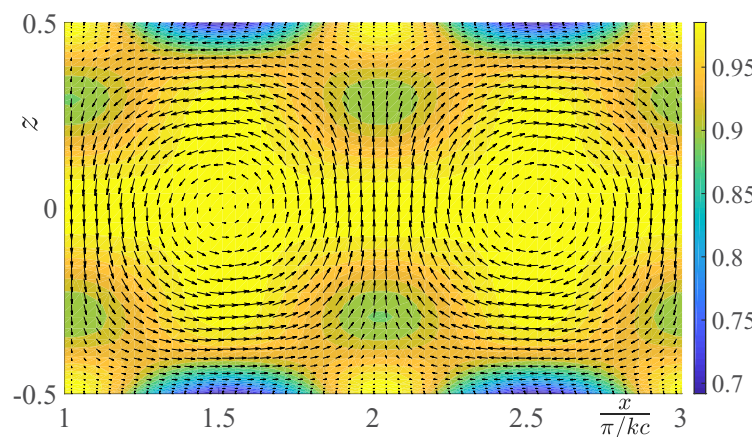

(a)

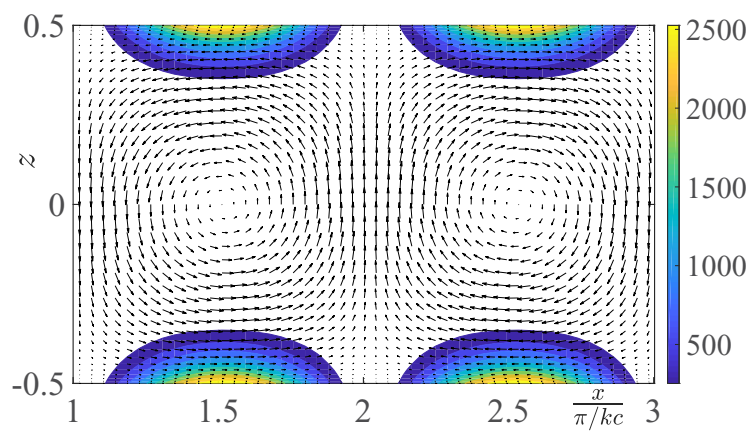

(b)

FIG. 6. Rolls. (a) Velocity vectors and viscosity field over a roll for a Carreau fluid with $\alpha=10^{-4}$ at $\epsilon=0.1$. (b) Distribution of $\dot{\gamma}_{x z}^{2}$. In the white zone, $\dot{\gamma}_{x z}^{2}<200$.

shrinks when $\Lambda$ decreases, which is understandable. Indeed, the thinner the slabs are, the weaker the thermal resistance is. Then the problem is closer to the case of perfectly conducting slabs where rolls are the preferred patterns. For $\Lambda>1$, the dependence of $\alpha_{S-R}$ with respect to $\Lambda$ is weak.

Finally, we observe that $\alpha_{S-R}$ increases as $\chi$ decreases. Poorly conducting slabs favor square patterns as shown in Refs. [10-12], so stronger shear-thinning effects are necessary so that rolls become the preferred planform. This last result is in agreement with Ref. [13].

\section{Flow structure, viscosity, and temperature fields}

In this section, features of the flow, temperature distribution, and shear-thinning effects on the viscosity field in a roll and a square solutions are studied for highly and poorly conducting slabs.

\section{Case of highly conducting walls: $\chi=100, \Lambda=1$}

The flow structure and the viscosity field for a roll solution are illustrated by Fig. 6 . The interior of the roll is practically isoviscous with $\mu \approx 1$. The viscosity is minimal at the walls where the shear-rate $\dot{\gamma}_{x z}$ is maximal. It is also weakly reduced at the four corners for a roll because of the elongational rate $\dot{\gamma}_{z z}=-\dot{\gamma}_{x x}$.

The distribution of the temperature perturbation over a roll with hot ascending flow and cold descending flow is illustrated

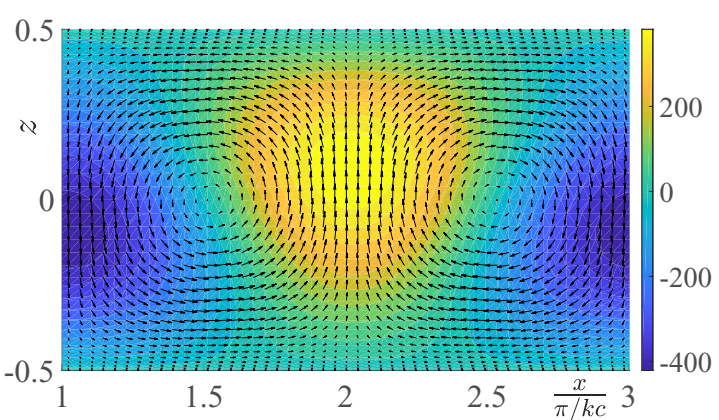

FIG. 7. Rolls. Contours of temperature perturbation over a roll with hot ascending flow and cold descending flow. Case of Carreau fluid with $\alpha=10^{-4}$ at $\epsilon=0.1$. by Fig. 7. It vanishes at the walls, because of the high value of the thermal conductivities ratio, $\chi$.

\section{Case of poorly conducting walls: $\chi=0.01, \Lambda=1$}

Because of the symmetries of the square solution, no fluid passes through the vertical diagonal planes and the vertical cell boundaries. The sides of the square have a length equal to $2 \pi / k_{c}$. The viscosity distribution and the velocity field in a horizontal plane close to the upper wall $(z=0.49)$ and in a vertical diagonal plane are illustrated by Fig. 8 for Carreau fluid with $\alpha=10^{-4}$ at $\epsilon=0.1$. The viscosity is minimal at location where the shear rate $\dot{\gamma}_{x z}$ and $\dot{\gamma}_{y z}$ [dark regions in Fig. 8(a)]. Contours of the temperature perturbation in a diagonal square cell section and in a lateral section that delimits the square cell are shown in Fig. 9. It is worth noting that the temperature perturbation does not vanish at the walls and the vertical thermal gradient is weak.

\section{E. Comparison between roll and square solutions for fixed $\chi$ and $\lambda$}

For fixed values $\chi$ and $\lambda$, velocity and viscosity fields are determined for roll and square solutions. It is observed that the maximum of shear rate and therefore the minimum of viscosity occurs for the stable pattern.

\section{F. Heat transfer}

The heat transfer through the horizontal fluid layer is described by the Nusselt number, $\mathrm{Nu}$, the ratio of the total heat to the purely conductive heat flux, i.e., when the fluid is at rest:

$$
\begin{aligned}
\mathrm{Nu} & =1-\left(\frac{\partial \bar{\theta}}{\partial z}\right)_{z=-1 / 2} \\
& =1-\left(|A|^{2}+|B|^{2}\right)\left(D G_{02}\right)_{z=-1 / 2},
\end{aligned}
$$

where $\left(|A|^{2}+|B|^{2}\right) G_{02}$ is the modification at the second order of the conductive temperature profile due to the interaction of the fundamental mode with its complex conjugate. The overbar denotes the average over one wavelength. Using the stationary 


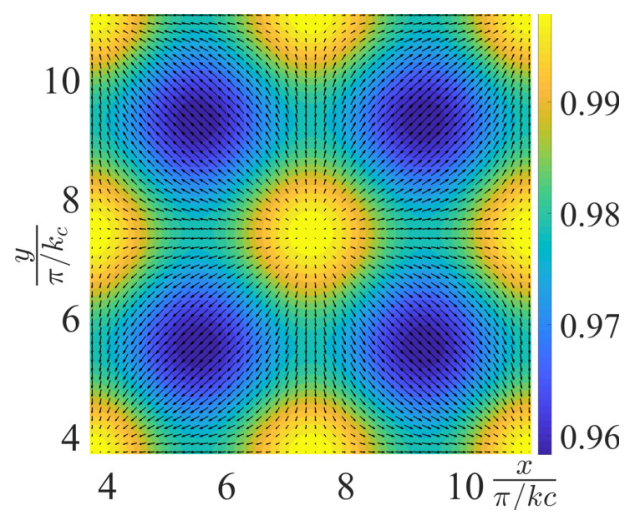

(a)

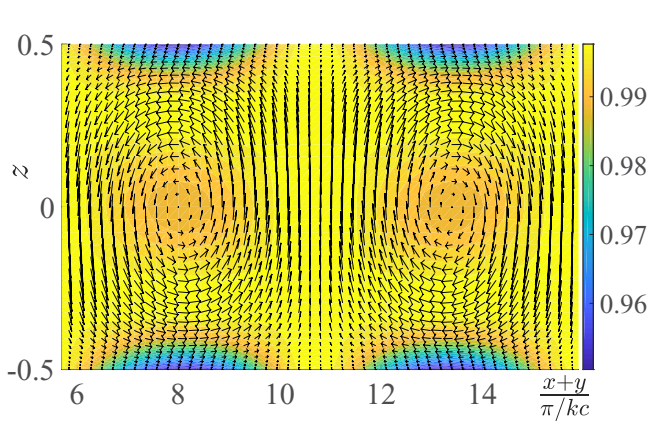

(b)

FIG. 8. Squares, $\chi=0.01, \Lambda=1$. Velocity vectors and viscosity field in (a) a horizontal plane close to the upper wall and (b) in a vertical diagonal plane. Case of Carreau fluid with $\alpha=10^{-4}$ at $\epsilon=0.1$.

solutions of the amplitude equations, one obtains

$$
\begin{array}{cc}
\mathrm{Nu}_{\mathrm{r}}=1-\frac{\epsilon}{\tau_{0} g_{1}}\left(D G_{02}\right)_{z=-1 / 2} & \text { for rolls, } \\
\mathrm{Nu}_{\mathrm{s}}=1-\frac{2 \epsilon}{\tau_{0}\left(g_{1}+\beta\right)}\left(D G_{02}\right)_{z=-1 / 2} & \text { for squares. }
\end{array}
$$

Figure 10 shows the variation of the Nusselt number as a function of the shear-thinning degree at $\epsilon=0.1, \chi=0.01$ and four different values of $\Lambda$. The Nusselt number increases with increasing shear-thinning effects in agreement with Refs. [6,33,34]. This is a consequence of the increase of the rolls amplitude. As expected, the Nusselt number decreases significantly with increasing the slab thickness. The difference between $\mathrm{Nu}$ rolls and $\mathrm{Nu}$ squares is small. Nevertheless, $\mathrm{Nu}$ is larger for the stable convective pattern in agreement with the maximum heat transfer principle: "The only stable solution is the one of maximum heat transport" $[35,36]$. This is also illustrated by Fig. 11, where Nu is represented as a function of $\epsilon$ for given $\alpha=4 \times 10^{-4}$ and $\chi=10^{-2}$. At $\Lambda=1$ and $\Lambda=0.1$ squares are stable and rolls are unstable, whereas at $\Lambda=0.01$, rolls are stable. Other principles can be considered to predict the stable pattern such the maximum entropy production or the maximum viscous dissipation [37,38]. Indeed, it can be shown that for a steady solution, $\operatorname{Ra}(\mathrm{Nu}-1)=\int_{\Omega} \tau_{i j} \dot{\gamma}_{i j} d \Omega$ [39], where $\Omega$ is a domain delimited by the top and bottom walls and one wavelength in the $x$ and $y$ directions. For shear-thinning fluids one can consider the principle of maximum viscosity reduction, as indicated in the previous section.

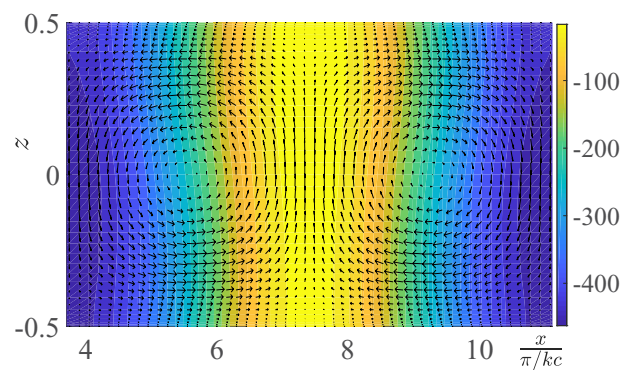

(a)

\section{SECONDARY INSTABILITIES}

Departing from the critical conditions, a band of wave numbers of width $O(\sqrt{\epsilon})$, centered on $k=k_{c}$ will now have a positive growth-rate. The wave packet centered on the most unstable wave number can be considered as a monochromatic wave, with complex amplitude modulated in space and time.

In a square lattice, and up to third order in the perturbations, the spatiotemporal evolution of the amplitudes is described by a set of two coupled Ginzburg-Landau equations derived by Newell and Whitehead [20] and Segel [40]:

$$
\begin{aligned}
& \frac{\partial A}{\partial t}=\frac{\epsilon}{\tau_{0}} A+\frac{\xi_{0}^{2}}{\tau_{0}}\left(\frac{\partial}{\partial x}-\frac{i}{2 k_{c}} \frac{\partial^{2}}{\partial y^{2}}\right)^{2} A-\left(g_{1}|A|^{2}+\beta|B|^{2}\right) A \\
& \frac{\partial B}{\partial t}=\frac{\epsilon}{\tau_{0}} B+\frac{\xi_{0}^{2}}{\tau_{0}}\left(\frac{\partial}{\partial y}-\frac{i}{2 k_{c}} \frac{\partial^{2}}{\partial x^{2}}\right)^{2} B-\left(g_{1}|B|^{2}+\beta|A|^{2}\right) B
\end{aligned}
$$

where the coherence length $\xi_{0}$ is defined by $\xi_{0}^{2}=$ $\frac{1}{2 \mathrm{Ra}_{\mathrm{c}}}\left(\frac{\partial^{2} \mathrm{Ra}}{\partial k^{2}}\right)_{\mathrm{Ra}_{\mathrm{c}}, k_{c}}$. It does not depend on rheological properties and can be calculated from the curve of the growth-rate $\sigma$ versus $\left(k-k_{c}\right)$. For $\chi \leqslant 10$, the coherence length $\xi_{0}$ varies between 0.375 and 0.415 . For $\chi>10$, we obtain $\xi_{0}=0.386$, which is in agreement with the literature [41].

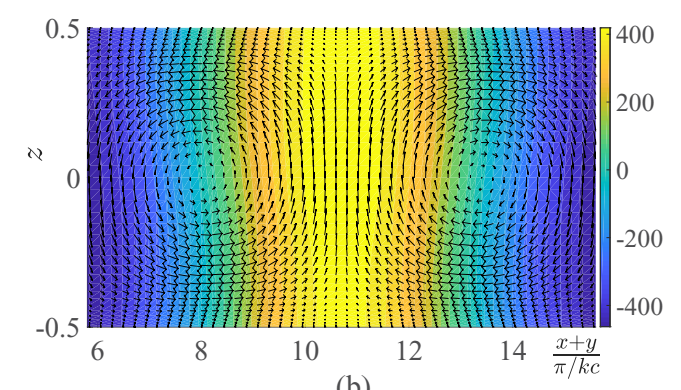

(b)

FIG. 9. Squares, $\chi=0.01, \Lambda=1$. Temperature distribution and velocity vectors in (a) a lateral section which delimits the square cell, $y=\pi / k_{c}$ and (b) in a vertical diagonal section. Case of Carreau fluid with $\alpha=10^{-4}$ at $\epsilon=0.1$. 


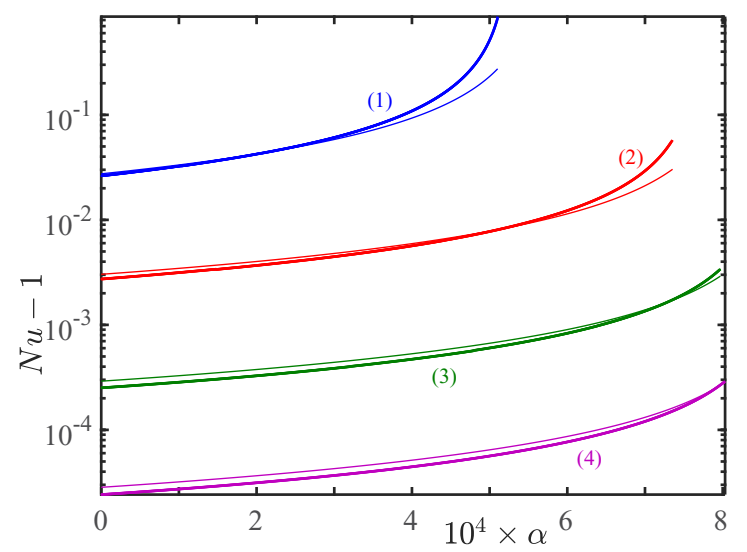

FIG. 10. The Nusselt number as a function of the shear-thinning degree $\alpha$ at $\epsilon=0.1, \chi=0.01, \operatorname{Pr}=10$ and different values of $\Lambda$ : (1) $\Lambda=0.01$, (2) $\Lambda=0.1$, (3) $\Lambda=1$ and (4) $\Lambda=10$. Thick lines, rolls; thin lines, squares.

\section{A. Stability of a roll modulated solution}

In the following, we consider first the case where rolls emerge at primary bifurcation $\left(\alpha>\alpha_{S-R}\right)$. We look for a stationary solution of the system Eqs. (51) and (52), of the form

$$
\begin{gathered}
A_{0}=R_{0} \exp (i q x) \quad \text { where } q=k-k_{c}, \\
B_{0}=0 .
\end{gathered}
$$

Substituting the above expressions into Eq. (51) leads to

$$
\begin{gathered}
R_{0}=\sqrt{\frac{\epsilon-\xi_{0}^{2} q^{2}}{g_{1} \tau_{0}} .} \quad \begin{array}{c}
\text { obtain after linearization and separating the } \\
\text { parts of the equations }
\end{array} \\
\frac{\partial r_{A}}{\partial t}=-2 g_{1} R_{0}^{2} r_{A}+\frac{\xi_{0}^{2}}{\tau_{0}}\left(\frac{\partial^{2} r_{A}}{\partial x^{2}}-2 q R_{0} \frac{\partial \Phi_{A}}{\partial x}+\frac{q}{k_{c}} \frac{\partial^{2} r_{A}}{\partial y^{2}}+\frac{R_{0}}{k_{c}} \frac{\partial^{3} \Phi_{A}}{\partial x \partial y^{2}}-\frac{1}{4 k_{c}^{2}} \frac{\partial^{4} r_{A}}{\partial y^{4}}\right), \\
\frac{\partial \Phi_{A}}{\partial t}=\frac{\xi_{0}^{2}}{\tau_{0}}\left(\frac{\partial^{2} \Phi_{A}}{\partial x^{2}}+\frac{2 q}{R_{0}} \frac{\partial r_{A}}{\partial x}+\frac{q}{k_{c}} \frac{\partial^{2} \Phi_{A}}{\partial y^{2}}-\frac{1}{k_{c} R_{0}} \frac{\partial^{3} r_{A}}{\partial x \partial y^{2}}-\frac{1}{4 k_{c}^{2}} \frac{\partial^{4} \Phi_{A}}{\partial y^{4}}\right), \\
\frac{\partial r_{B}}{\partial t}=\left(g_{1}-\beta\right) R_{0}^{2} r_{B}+q^{2} \frac{\xi_{0}^{2}}{\tau_{0}} r_{B}+\frac{\xi_{0}^{2}}{\tau_{0}}\left(\frac{\partial^{2} r_{B}}{\partial y^{2}}-\frac{1}{4 k c^{2}} \frac{\partial^{4} r_{B}}{\partial x^{4}}\right) .
\end{gathered}
$$

Equation associated with $\partial \Phi_{B} / \partial t$ does not contain linear terms so $\Phi_{B}$ does not intervene at the first order. Using normal mode decomposition, i.e., $\Psi(x, y, t)=\widetilde{\Psi} \exp \left[\sigma t+i\left(Q_{1} x+Q_{2} y\right)\right]$, where $\Psi$ stands for $r_{A}, \Phi_{A}$ and $r_{B}$, an eigenvalue problem is derived:

$$
\mathbf{L X}=\sigma \mathbf{X}
$$

where $\mathbf{X}=\left(\widetilde{r_{A}}, \widetilde{\Phi_{A}}, \widetilde{r_{B}}\right)^{T}$ is the eigenvector, $\sigma$ the eigenvalue, and $\boldsymbol{L}$ a $3 \times 3$ square matrix arising from Eqs. (58)(60). Note that the eigenvalue problem corresponding to Eq. (60) can be solved independently from the whole system.

We consider the long wavelength limit where $Q_{1} \rightarrow 0$ and $Q_{2} \rightarrow 0$. In that case, the relevant eigenvalues of the former system are given by

$$
\begin{gathered}
\sigma_{1}=-2 g_{1} R_{0}^{2}+O\left(Q_{1}\right), \\
\sigma_{2}=-\frac{\xi_{0}^{2}}{\tau_{0}} Q_{1}^{2}\left[1-\frac{2 \xi_{0}^{2}}{\tau_{0}} \frac{q^{2}}{g_{1} R_{0}^{2}}\right]-\frac{\xi_{0}^{2}}{\tau_{0}} \frac{q Q_{2}^{2}}{k_{c}}+O\left(Q_{1}^{2} Q_{2}^{2}\right),
\end{gathered}
$$

$$
\sigma_{3}=\frac{\xi_{0}^{2}}{\tau_{0}} q^{2}+\left(g_{1}-\beta\right) R_{0}^{2}+O\left(Q_{1}^{4}, Q_{2}^{2}\right) .
$$

The eigenvector $\left(\widetilde{r}_{A}, \widetilde{\Phi}_{A}\right)$ associated with the first eigenvalue $\sigma_{1}=-2 g_{1} R_{0}^{2}<0$ is $\left.\left(O\left(1 / Q_{1}\right), 1\right)\right)$. Therefore, $\sigma_{1}$ describes the relatively rapid relaxation of the amplitude perturbation $r_{A}$ to its equilibrium value. The eigenvector $\left(\widetilde{r}_{A}, \widetilde{\Phi}_{A}\right)$ associated with $\sigma_{2}$ is $\left(O\left(Q_{1}\right), 1\right)$. The second eigenvalue describes the evolution of the phase perturbation $\Phi_{A}$. 
The third root $\sigma_{3}$ describes the evolution of rolls growing perpendicularly to the original ones.

The eigenvalue $\sigma_{2}$ can also be derived using the phase approximation. This approach described in Refs. [32,42] relies on the fact that the amplitude $r_{A}$ relaxes quickly with time, it can be considered to be adiabatically slaved to the phase $\Phi_{A}$. This comes down to writing $\partial r_{A} / \partial t=0$. Furthermore, in the long wavelength limit, spatial derivatives are very small compared to the variables themselves. Therefore, the amplitude $r_{A}$ is approximately given by its adiabatic value,

$$
r_{A}=-\frac{q \xi_{0}^{2}}{g_{1} R_{0} \tau_{0}} \frac{\partial \Phi_{A}}{\partial x} .
$$

This expression is substituted in Eq. (59) to determine the evolution of $\phi_{A}$. A phase-diffusion equation is then derived:

$$
\frac{\partial \Phi_{A}}{\partial t}=D_{\|} \frac{\partial^{2} \Phi_{A}}{\partial x^{2}}+D_{\perp} \frac{\partial^{2} \Phi_{A}}{\partial y^{2}} .
$$

The longitudinal $D_{\|}$and transverse $D_{\perp}$ phase diffusion coefficients are given by

$$
D_{\|}=\frac{\xi_{0}^{2}}{\tau_{0}}\left(1-\frac{2 q^{2}}{g_{1} R_{0}^{2}} \frac{\xi_{0}^{2}}{\tau_{0}}\right) \quad \text { and } \quad D_{\perp}=\frac{\xi_{0}^{2}}{\tau_{0}} \frac{q}{k_{c}} .
$$

Equation (66) shows that a perturbation of the wave number leads to a readjustment of the system through a phase diffusion process. The eigenvalue that stems from Eq. (66) is the same as $\sigma_{2}$.

\section{Eckhaus instability}

For perturbations that vary only in the $x$ direction $\left(Q_{2}=0\right)$, the eigenvalue $\sigma_{2}$ Eq. (63) reduces to

$$
\sigma_{2}=-Q_{1}^{2} D_{\|}+O\left(Q_{1}^{4}\right)
$$

For positive longitudinal phase-diffusion coefficient $D_{\|}, \sigma_{2}$ is negative, the perturbation is damped and the roll solution Eq. (53) is stable. Using Eq. (55), the stability is satisfied if

$$
\epsilon>\epsilon_{E}=3 q^{2} \xi_{0}^{2}
$$

where the subscript E means "Eckhaus." Note that this instability does not depend on the rheological parameters.

\section{Zigzag instability}

For perturbations that vary only in the $y$ direction $\left(Q_{1}=0\right)$, the eigenvalue $\sigma_{2}$ Eq. (63) reduces to

$$
\sigma_{2}=-Q_{2}^{2} D_{\perp}+O\left(Q_{2}^{4}\right)
$$

For negative $D_{\perp}$, i.e., when $q$ is negative $\left(k<k_{c}\right)$, rolls at wavelength greater than the critical one, the eigenvalue $\sigma_{2}$ is positive, the perturbation is amplified and the roll solution Eq. (53) is unstable. In this case, the rolls will saturate into bends that decrease the wavelength.

\section{Cross-Roll instability}

The eigenvalue $\sigma_{3}$ Eq. (64) corresponds to the cross-roll (CR) instability. The system is CR stable if $\sigma_{3}<0$. Using Eq. (55), the system is CR stable if

$$
\epsilon>\epsilon_{\mathrm{CR}}=\frac{\beta}{\beta-g_{1}} q^{2} \xi_{0}^{2},
$$

where the subscript CR means "cross-roll." When $\epsilon<\epsilon_{\mathrm{CR}}$, the stationary roll solution Eq. (53) becomes unstable: new rolls expand perpendicularly. It can be shown straightforwardly that the cross-roll is a more restrictive instability than the Eckhaus instability, when

$$
\beta<\frac{3}{2} g_{1}
$$

-For a Newtonian fluid at $\operatorname{Pr}=10$ and in the case of perfectly conducting walls, $\beta / g_{1}=1.242$. Decreasing the conductivity of the wall, $\chi$, will decrease $\left(\beta / g_{1}\right)$ and will give a narrow band of stable rolls. The width of this band vanishes as the singularity $\beta / g_{1} \rightarrow 1^{+}$is approached.

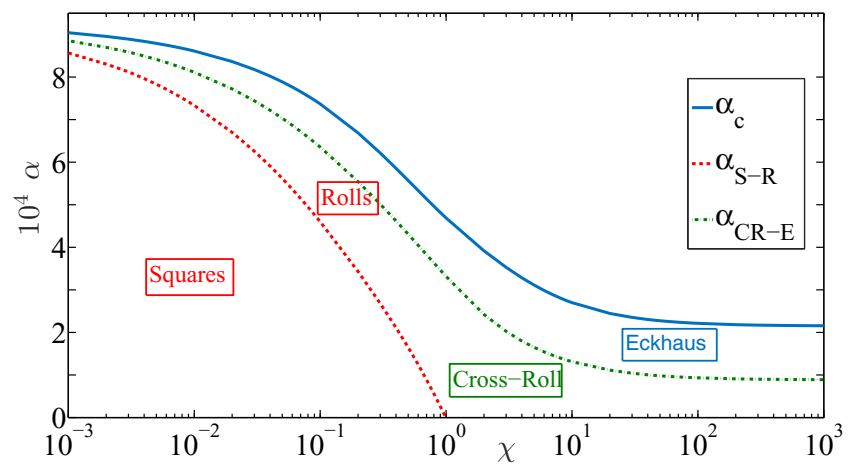

(a) $\Lambda=1$

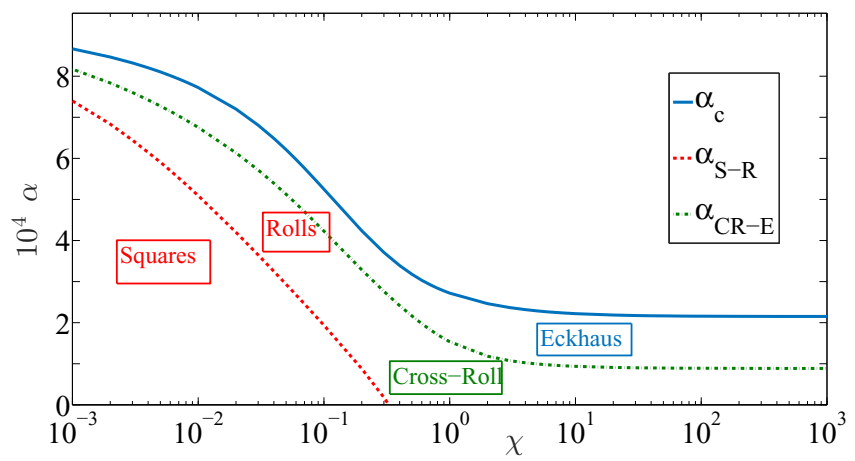

(b) $\Lambda=0.1$

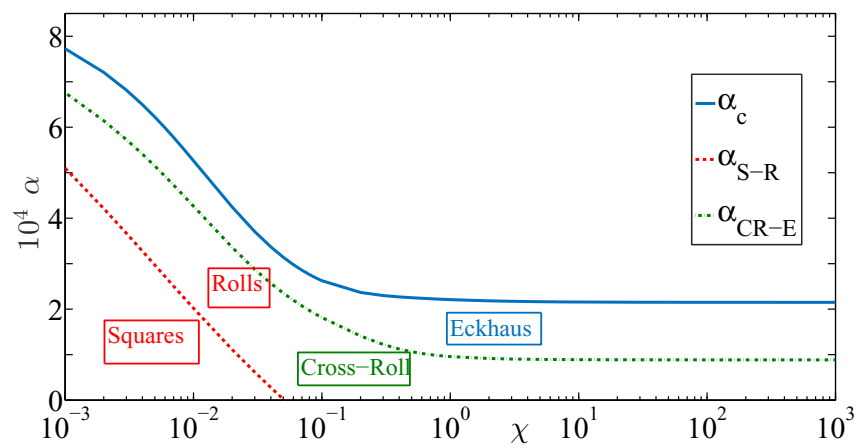

(c) $\Lambda=0.01$

FIG. 12. Boundary between Eckhaus and cross-roll instabilities for different slabs' thicknesses $\Lambda$ : (a) $\Lambda=1$ and (b) $\Lambda=0.1$, (c) $\Lambda=0.01$. 
-For a shear-thinning fluid, $\beta / g_{1}$ increases with increasing shear-thinning effects. Combining Eq. (72) with Eqs. (43) and (44), we can define a shear-thinning degree $\alpha_{\mathrm{CR}-E}$, below which cross-roll is the more restrictive instability:

$$
\alpha_{\mathrm{CR}-E}=\frac{3 g_{1}^{N}-2 \beta^{N}}{3 g_{1}^{n N}-2 \beta^{n N}} .
$$

For $\alpha>\alpha_{\mathrm{CR}-E}$, the Eckhaus instability takes over as the most restrictive of the two. Figure 12 shows the variation of $\alpha_{\mathrm{CR}-E}$ as a function of the conductivity of the walls and for different values of the thickness $\Lambda$. We have also represented the boundaries $\alpha_{S-R}$ and $\alpha_{c}$ (limit of subcritical bifurcation). As expected, $\alpha_{\mathrm{CR}-E}$ increasing with decreasing $\chi$. In Fig. 13, we have represented in the plane $(k, \mathrm{Ra})$ the curves that delimit the stability domain of rolls with respect to (i) cross-roll instability for different $\alpha$ and (ii) Eckhaus instability (which is independent of $\alpha$ ). With increasing shearthinning effects $\left(\beta / g_{1}\right.$ increases), the CR stability boundary enlarges and becomes less restrictive than Eckhaus instability for $\alpha>\alpha_{\mathrm{CR}-E}$.

\section{B. Stability of a square-modulated solution}

In the case where the convection starts with perfect square patterns, a stationary solution is given by

$$
\begin{gathered}
A_{0}(x)=R_{0} \exp (i q x) \quad \text { where } q=k-k_{c}, \\
B_{0}(y)=R_{0} \exp (i q y) .
\end{gathered}
$$

Replacing these expressions in Eqs. (51) and (52) leads to

$$
\begin{gathered}
R_{0}=\sqrt{\frac{\epsilon-\xi_{0}^{2} q^{2}}{\left(g_{1}+\beta\right) \tau_{0}}} \quad \text { (76) } \begin{array}{r}
\text { expressions, Eqs. (77) and (78) into Eqs. (51) } \\
\text { after linearization to }
\end{array} \\
\frac{\partial r_{A}}{\partial t}=-2 g_{1} R_{0}^{2} r_{A}-2 \beta R_{0}^{2} r_{B}+\frac{\xi_{0}^{2}}{\tau_{0}}\left(\frac{\partial^{2} r_{A}}{\partial x^{2}}-2 q R_{0} \frac{\partial \Phi_{A}}{\partial x}+\frac{q}{k_{c}} \frac{\partial^{2} r_{A}}{\partial y^{2}}+\frac{R_{0}}{k_{c}} \frac{\partial^{3} \Phi_{A}}{\partial x \partial y^{2}}-\frac{1}{4 k_{c}^{2}} \frac{\partial^{4} r_{A}}{\partial y^{4}}\right), \\
\frac{\partial \Phi_{A}}{\partial t}=\frac{\xi_{0}^{2}}{\tau_{0}}\left(\frac{\partial^{2} \Phi_{A}}{\partial x^{2}}+\frac{2 q}{R_{0}} \frac{\partial r_{A}}{\partial x}-\frac{1}{k_{c} R_{0}} \frac{\partial^{3} r_{A}}{\partial x \partial y^{2}}+\frac{q}{k_{c}} \frac{\partial^{2} \Phi_{A}}{\partial y^{2}}-\frac{1}{4 k_{c}^{2}} \frac{\partial^{4} \Phi_{A}}{\partial y^{4}}\right), \\
\frac{\partial r_{B}}{\partial t}=-2 g_{1} R_{0}^{2} r_{B}-2 \beta R_{0}^{2} r_{A}+\frac{\xi_{0}^{2}}{\tau_{0}}\left(\frac{\partial^{2} r_{B}}{\partial y^{2}}-2 q R_{0} \frac{\partial \Phi_{B}}{\partial y}+\frac{q}{k_{c}} \frac{\partial^{2} r_{B}}{\partial x^{2}}+\frac{R_{0}}{k_{c}} \frac{\partial^{3} \Phi_{B}}{\partial x^{2} \partial y}-\frac{1}{4 k_{c}^{2}} \frac{\partial^{4} r_{B}}{\partial x^{4}}\right), \\
\frac{\xi_{0}^{2}}{\tau_{0}}\left(\frac{\partial^{2} \Phi_{B}}{\partial y^{2}}+\frac{2 q}{R_{0}} \frac{\partial r_{B}}{\partial y}-\frac{1}{k_{c} R_{0}} \frac{\partial^{3} r_{B}}{\partial x^{2} \partial y}+\frac{q}{k_{c}} \frac{\partial^{2} \Phi_{B}}{\partial x^{2}}-\frac{1}{4 k_{c}^{2}} \frac{\partial^{4} \Phi_{B}}{\partial x^{4}}\right) .
\end{gathered}
$$

Using a normal modes decomposition, i.e., $\Psi(x, y, t)=$ $\tilde{\Psi} \exp \left[\sigma t+i\left(Q_{1} x+Q_{2} y\right)\right]$, where $\Psi$ stands for $r_{A}, \Phi_{A}, r_{B}, \Phi_{B}$, the following eigenvalue problem is derived:

$$
\mathbf{M X}=\sigma \mathbf{X}
$$

In Eq. (83), $\mathbf{X}=\left(\widetilde{r_{A}}, \widetilde{\Phi_{A}}, \widetilde{r_{B}}, \widetilde{\Phi_{B}}\right)^{T}$ is the eigenvector, $\sigma$ the eigenvalue and $\mathbf{M}$ the $4 \times 4$ square matrix arising from Eqs. (79)-(82). The eigenvalues and eigenvectors can be determined numerically using Matlab. Examples of results are shown in Appendix A.

Actually, we are particularly interested by the long wavelength limit approach, i.e., $Q_{1} \rightarrow 0$ and $Q_{2} \rightarrow 0$. In this approach, the eigenvalues $\sigma_{1}$ and $\sigma_{2}$ associated with the amplitudes $r_{A}$ and $r_{B}$, respectively, are given by

$$
\begin{aligned}
& \sigma_{1}=-2 R_{0}^{2}\left(g_{1}+\beta\right)+O\left(Q_{1}^{2}, Q_{2}^{2}\right) \\
& \sigma_{2}=-2 R_{0}^{2}\left(g_{1}-\beta\right)+O\left(Q_{1}^{2}, Q_{2}^{2}\right) .
\end{aligned}
$$


Since, in this case $\beta$ and $g_{1}$ are positive and $\beta<g_{1}$, the amplitude modes $r_{A}$ and $r_{B}$ decrease quickly with time and can be considered adiabatically slaved to the phase modes $\Phi_{A}$ and $\Phi_{B}$. They can be approximated by their adiabatic values:

$$
\begin{aligned}
& r_{A}=\frac{q}{R_{0}\left(g_{1}^{2}-\beta^{2}\right)} \frac{\xi_{0}^{2}}{\tau_{0}}\left(\beta \frac{\partial \Phi_{B}}{\partial y}-g_{1} \frac{\partial \Phi_{A}}{\partial x}\right), \\
& r_{B}=\frac{q}{R_{0}\left(g_{1}^{2}-\beta^{2}\right)} \frac{\xi_{0}^{2}}{\tau_{0}}\left(\beta \frac{\partial \Phi_{A}}{\partial x}-g_{1} \frac{\partial \Phi_{B}}{\partial y}\right) .
\end{aligned}
$$

Substituting these expressions in Eqs. (80) and (82) leads to the following diffusion equations of phases $\Phi_{A}$ and $\Phi_{B}$ :

$$
\begin{aligned}
\frac{\partial \Phi_{A}}{\partial t} & =D_{\|} \frac{\partial^{2} \Phi_{A}}{\partial x^{2}}+D_{\perp} \frac{\partial^{2} \Phi_{A}}{\partial y^{2}}+D_{x y} \frac{\partial^{2} \Phi_{B}}{\partial x \partial y}, \\
\frac{\partial \Phi_{B}}{\partial t} & =D_{\|} \frac{\partial^{2} \Phi_{B}}{\partial y^{2}}+D_{\perp} \frac{\partial^{2} \Phi_{B}}{\partial x^{2}}+D_{x y} \frac{\partial^{2} \Phi_{A}}{\partial y \partial x},
\end{aligned}
$$

where the coefficients phase-diffusion have the following expressions:

$$
\begin{aligned}
D_{\|} & =\frac{\xi_{0}^{2}}{\tau_{0}}\left(1-\frac{2 q^{2} g_{1}}{R_{0}^{2}\left(g_{1}^{2}-\beta^{2}\right)} \frac{\xi_{0}^{2}}{\tau_{0}}\right) ; \quad D_{\perp}=\frac{\xi_{0}^{2}}{\tau_{0}} \frac{q}{k_{c}} ; \\
D_{x y} & =\frac{2 q^{2} \beta}{R_{0}^{2}\left(g_{1}^{2}-\beta^{2}\right)}\left(\frac{\xi_{0}^{2}}{\tau_{0}}\right)^{2} .
\end{aligned}
$$

Using normal mode decomposition, an eigenvalue problem is derived. The eigenvalues are

$$
\begin{aligned}
& \sigma_{3}=-\left(Q_{1}^{2} D_{\|}+Q_{2}^{2} D_{\perp}\right)-Q_{1} Q_{2} D_{x y} \\
& \sigma_{4}=-\left(Q_{1}^{2} D_{\|}+Q_{2}^{2} D_{\perp}\right)+Q_{1} Q_{2} D_{x y} .
\end{aligned}
$$

\section{Phase instabilities: case where $Q_{1}=Q_{2}$}

Considering the case where $Q_{1}=Q_{2}=Q$ and a long wavelength limit, i.e., $Q \rightarrow 0$, the eigenvalues Eqs. (90) reduce to

$$
\begin{aligned}
\sigma_{3} & =-Q^{2}\left[D_{\|}+D_{\perp}+D_{x y}\right] \\
& =\frac{\xi_{0}^{2}}{\tau_{0}} Q^{2}\left[2 \frac{\xi_{0}^{2}}{\tau_{0}} \frac{q^{2}}{R_{0}^{2}\left(g_{1}+\beta\right)}-\frac{k_{c}+q}{k_{c}}\right], \\
\sigma_{4} & =-Q^{2}\left[D_{\|}+D_{\perp}-D_{x y}\right] \\
& =\frac{\xi_{0}^{2}}{\tau_{0}} Q^{2}\left[2 \frac{\xi_{0}^{2}}{\tau_{0}} \frac{q^{2}}{R_{0}^{2}\left(g_{1}-\beta\right)}-\frac{k_{c}+q}{k_{c}}\right] .
\end{aligned}
$$

\section{Square Eckhaus instability}

The eigenvalue $\sigma_{3}$ Eq. (91) states that the system is stable provided that

$$
\epsilon>\epsilon_{\mathrm{SE}}=\left(\frac{3 k_{c}+q}{k_{c}+q}\right) \xi_{0}^{2} q^{2},
$$

where $\epsilon_{\mathrm{SE}}$ is the boundary of the square Eckhaus instability. When $q \ll k_{c}$, the universal expression $\epsilon=3 \xi_{0}^{2} q^{2}$ is recovered. In the phase approximation, the eigenvector corresponding to $\sigma_{3}$ is $(1,1)$. The wave numbers in the $x$ and $y$ directions evolve in the same way. Note that like for two-dimensional rolls, $\epsilon_{\mathrm{SE}}$ does not depend on the rheological parameters.

\section{Rectangular Eckhaus instability}

The eigenvalue $\sigma_{4}$ Eq. (92) states that the system is stable provided that

$$
\epsilon>\epsilon_{\mathrm{RI}}=\xi_{0}^{2} q^{2}\left[1+2 \frac{g_{1}+\beta}{g_{1}-\beta} \frac{k_{c}}{k_{c}+q}\right] .
$$

When $q \ll k_{c}$, we recover the expression given by Holmedal [19] and Hoyle [18], i.e., $\epsilon_{\mathrm{RI}}=\left(3 g_{1}+\beta\right) /\left(g_{1}-\beta\right)$. An eigenvector associated with $\sigma_{4}$ Eq. (92) is $(1,-1)$; therefore, the wave numbers in the $x$ and $y$ directions do not have the same time evolution. Hoyle [18] denoted $\epsilon_{\mathrm{RI}}$ Eq. (94) as rectangular Eckhaus instability. Another point of view was given by Holmedal [19]. According to this author, since the eigenvector is $(-1,1)$, one of the two rolls will grow at the expense of the other at a particular horizontal location. The decreasing mode will be the growing one at another location. This instability is denoted by Holmedal [19] as "long wavelength cross-roll instability." Concerning the influence of the rheological parameters, it can be shown straightforwardly that $\epsilon_{\mathrm{RI}}$ boundary shrinks with increasing shear-thinning effects.

\section{Phase instabilities: Case where either $Q_{1}$ or $Q_{2}$ is zero}

In the case where either $Q_{1}$ or $Q_{2}$ is zero, the phase Eqs. (87) and $(88)$ reduce to

$$
\frac{\partial \Phi_{A}}{\partial t}=D_{\|} \frac{\partial^{2} \Phi_{A}}{\partial x^{2}} \quad \text { and } \quad \frac{\partial \Phi_{B}}{\partial t}=D_{\perp} \frac{\partial^{2} \Phi_{B}}{\partial x^{2}} .
$$

The eigenvalues $\sigma_{3}$ and $\sigma_{4}$ of phase-diffusion Eqs. (95) are then

$$
\sigma_{3}=-Q_{1}^{2} D_{\|} \quad \text { and } \sigma_{4}=-Q_{1}^{2} D_{\perp}
$$

\section{E. Zigzag instability}

The eigenvalue $\sigma_{4}$ Eq. (96), which is independent of the rheological parameters, causes an instability of the squares if $q<0$, i.e., $k<k_{c}$. This is similar to the condition for zigzag instability of the rolls.

\section{F. 2D Eckhaus instability}

The eigenvalue $\sigma_{3}$ Eq. (96) leads to another phase instability boundary given by

$$
\epsilon_{2 \mathrm{DE}}=\xi_{0}^{2} q^{2}\left(\frac{3 g_{1}-\beta}{g_{1}-\beta}\right),
$$

which can be considered as a 2D Eckhaus instability [18].

Finally, it can be shown straightforwardly that $\epsilon_{\mathrm{RI}}>\epsilon_{2 \mathrm{DE}}>$ $\epsilon_{\mathrm{SE}}$ when $\beta<g_{1}$. Therefore, the stability boundaries of the square pattern are given by the condition $k>k_{c}$ (zigzag instability) and Eq. (94), i.e., Eckhaus rectangular instability. Figure 14 depicts the domain of stability of the square stationary solution for different values of the shear thinning degree $\alpha$. The range of stable wave number for square patterns decreases with increasing shear-thinning effects, in contrast with the case of roll patterns. 


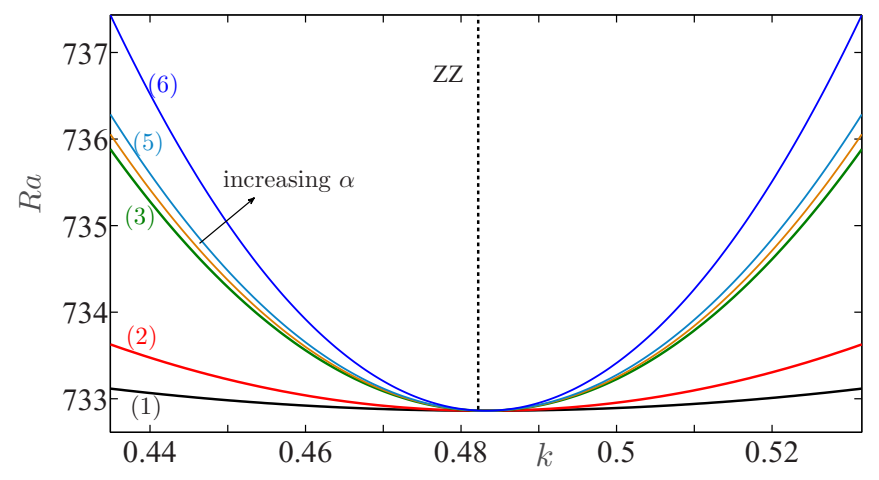

FIG. 14. Influence of shear thinning effects on stability boundaries of squares as a function of the Rayleigh number $\mathrm{Ra}$ and the wave number $k$ for $\Lambda=1, \operatorname{Pr}=10$ and $\chi=10^{-3}$. (1) Marginal stability curve, (2) Square Eckhaus boundary, (3) RI boundary for a Newtonian fluid, (4) RI boundary for Carreau fluid with $\alpha=0.5 \times \alpha_{S-R}=$ $4.25 \times 10^{-4},(5)=\alpha=0.75 \times \alpha_{S-R}=6.37 \times 10^{-4}$, and (6) $\alpha=$ $0.5 \times \alpha_{S-R}=7.65 \times 10^{-4}$. (ZZ) is the zigzag boundary.

\section{NUMERICAL SOLUTIONS OF AMPLITUDE EQUATIONS}

\section{A. Numerical simulation}

The secondary instabilities described in the previous section are studied here by solving numerically the Ginzburg-Landau equations. For the numerical integration of Eqs. (51) and (52), we employed a Fourier pseudospectral method on a square mesh with periodic boundary conditions. The square domain $[-L / 2, L / 2] \times[-L / 2, L / 2]$ is discretized into $N \times$ $N$ uniformly spaced grid points $M_{\ell p}=\left(x_{\ell}, y_{p}\right)$ with $x_{\ell}=$ $-L / 2+\ell \Delta x$ (similarly for $\left.y_{p}\right), \Delta x=\Delta y=L / N$ and $N$ even. Given $A_{M_{\ell p}}=A_{\ell p}, \ell, p=1,2, \ldots N$ ( similarly for $B_{\ell p}$ ), the 2D Discrete Fourier Transform (2DFT) is defined as

$$
\begin{aligned}
& \hat{A}_{k_{x} k_{y}}=\Delta x \Delta y \sum_{\ell=1}^{N} \sum_{p=1}^{N} A_{\ell p} e^{-i\left(k_{x} x_{\ell}+k_{y} y_{p}\right)}, \\
& k_{x}, k_{y}=\frac{2 \pi}{L}\left(\frac{-N}{2}, \ldots, \frac{N}{2}-1\right) .
\end{aligned}
$$

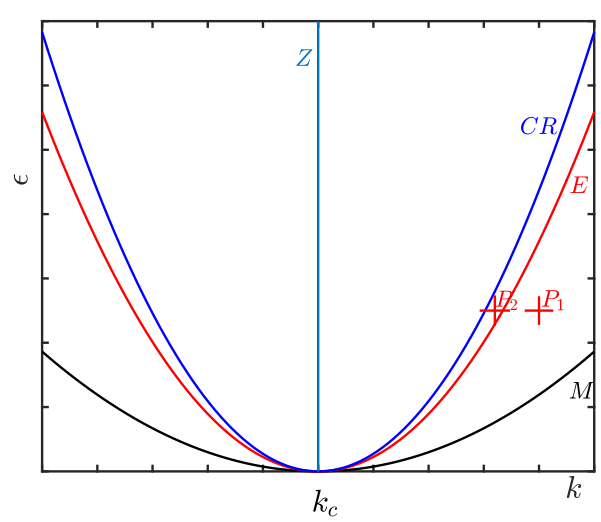

(a)
Leaving the time stepping in Fourier space, gives the following system of ODEs:

$$
\begin{aligned}
\frac{d}{d t} \hat{A}_{k_{x} k_{y}}= & {\left[\frac{\epsilon}{\tau_{0}}-\frac{\xi_{0}^{2}}{\tau_{0}}\left(k_{x}^{2}+\frac{k_{x} k_{y}^{2}}{k_{c}}+\frac{k_{y}^{4}}{4 k_{c}^{2}}\right)\right] \hat{A}_{k_{x} k_{y}} } \\
& -\mathcal{N}_{1, k_{x} k_{y}}(A, B), \\
\frac{d}{d t} \hat{B}_{k_{x} k_{y}}= & {\left[\frac{\epsilon}{\tau_{0}}-\frac{\xi_{0}^{2}}{\tau_{0}}\left(k_{y}^{2}+\frac{k_{y} k_{x}^{2}}{k_{c}}+\frac{k_{x}^{4}}{4 k_{c}^{2}}\right)\right] \hat{B}_{k_{x} k_{y}} } \\
& -\mathcal{N}_{2, k_{x} k_{y}}(A, B),
\end{aligned}
$$

with Fourier transformed initial conditions. The nonlinear terms $\mathcal{N}_{1, k_{x} k_{y}}$ and $\mathcal{N}_{2, k_{x} k_{y}}$ are evaluated in physical space and then transformed to Fourier space:

$$
\begin{aligned}
\mathcal{N}_{1, k_{x} k_{y}}(A, B)= & -g_{1} \mathcal{F}\left(|A(x, y, t)|^{2} A(x, y, t)\right) \\
& -\beta \mathcal{F}\left(|B(x, y, t)|^{2} A(x, y, t)\right),
\end{aligned}
$$

and

$$
\begin{aligned}
\mathcal{N}_{2, k_{x} k_{y}}(A, B)= & -g_{1} \mathcal{F}\left(|B(x, y, t)|^{2} B(x, y, t)\right) \\
& -\beta \mathcal{F}\left(|A(x, y, t)|^{2} B(x, y, t)\right),
\end{aligned}
$$

where $\mathcal{F}$ designates the $2 \mathrm{D}$ discrete Fourier transform. For the temporal discretization, the time domain $\left[0, t_{\max }\right]$ is discretized with equal time step of width $\Delta t$ as $t_{m}=$ $m \Delta t, m=0,1,2 \ldots$. Exponential Time Differencing method of second order (ETD2) proposed by Cox and Matthews [43] is used. Additional details can be found in Ref. [44]. The pseudospectral method is implemented in Matlab. Finally, to check the convergence, several simulations are carried out with increasing numbers of grid points and refining the time step. The stability properties of ETD2 are given in Appendix B.

\section{B. Numerical results}

\section{Instability of a roll solution}

Integration of the amplitude Eqs. (51) and (52) is performed at some representative points shown in Fig. 15 by the symbol $(+)$, for two cases: (a) low or moderate shear-thinning effects and (b) high shear-thinning effects. The position of these points

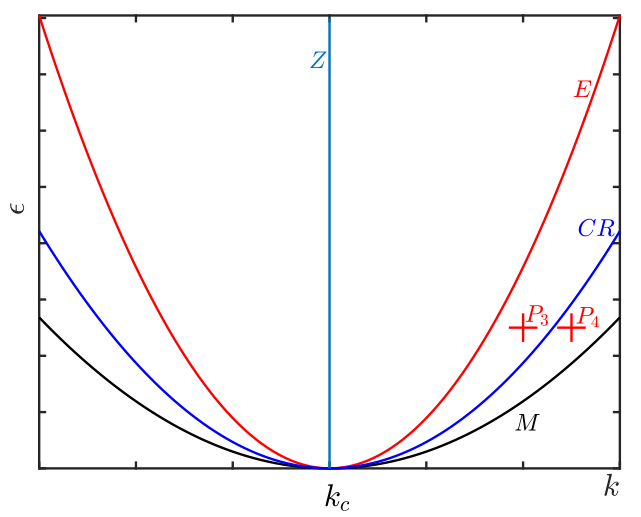

(b)

FIG. 15. Rolls: Points $P_{1}, P_{2}, P_{3}, P_{4}$, where numerical simulations were performed. (a) Low or moderate shear thinning effects, (b) strong shear thinning effects. (M) Marginal stability curve, (E) Eckhaus boundary, (CR) cross-roll boundary, (Z) zigzag boundary. 
(a)

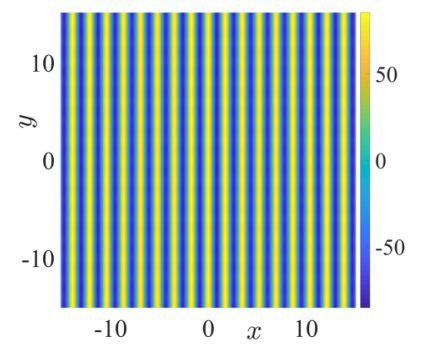

(c)

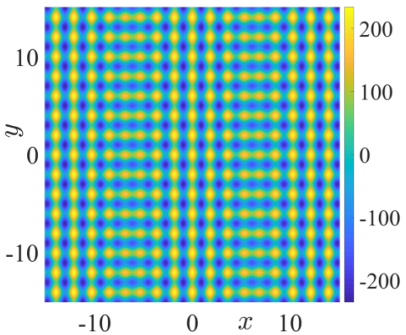

(b)

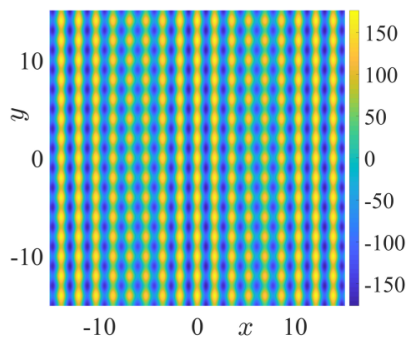

(d)

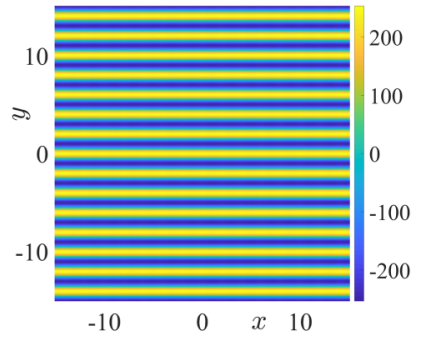

FIG. 16. Four subsequent stages in the simulation of the coupled amplitude Eqs. (51) and (52), evolving from CR unstable roll to CR stable roll. (a) $t=0$, (b) $t=4.5$, (c) $t=6$, (d) $t=21$.

with respect to Eckhaus (E) and cross-roll (CR) boundaries is clearly indicated.

Cross-roll instability. For moderate shear-thinning effects, i.e., $\alpha<\alpha_{\mathrm{CR}-E}$ given by Eq. (73), the stability diagram (Fig. 13) indicates that the region of stable rolls is bounded by the CR instability. For a given Rayleigh number not too far above the critical value, roll solution with a wave number $k$ outside the CR boundary is either CR unstable [point $P_{2}$ in Fig. 15(a)] or Eckhaus and CR unstable [point $P_{1}$ in Fig. 15(a) ] if $k$ is sufficiently large. At the point $P_{1}$, we have the following parameters: $q=0.4, \epsilon=0.05, \alpha=5 \times 10^{-5}, \Lambda=$ 1 , and $\chi=100$. The convective pattern is Eckhaus and CR unstable, but CR is dominant. This is indeed what happens as illustrated in Fig. 16, where the planform function, $f(x, y, t)=$ $A(x, y, t) e^{i k_{c} x}+B(x, y, t) e^{i k_{c} y}+$ c.c., is represented. Initially, we have a uniform set of rolls, $A=R_{0} \exp (i q x)$ and $B=0$, with $R_{0}$ given by Eq. (55). Small random perturbations have been added to this initial stationary solution. Due to the CR instability, perpendicular rolls grow and the initial rolls decay until the initial rolls with their too short wavelength are taken over by the perpendicular cross-roll with a wave number close to the critical value. Similar results are obtained at point $P_{2}$ and therefore are not represented.

Eckhaus instability. For sufficiently strong shear-thinning effects, i.e., $\alpha>\alpha_{\mathrm{CR}-E}$, a roll solution with a wave number outside Eckhaus boundary (curve 2, Fig. 13), can be either Eckhaus unstable and CR stable ([ $P_{3}$ in Fig. 15(b)] or Eckhaus and $\mathrm{CR}$ unstable $\left[P_{4}\right.$ in Fig. 15(b)]. For these two situations, Eckhaus instability mechanism is dominant. This is illustrated by Figs. 17 and 18 where the planform function $f(x, y, t)$ is represented. At point $P_{3}$, the initial state is a uniform roll solution with $q=k-k_{c}=0.4, \epsilon=0.05$, and $\alpha=1.610^{-4}$. It is in CR-stable and in Eckhaus unstable region, where the pattern wavelength is too short. A small random perturbation is added in the $x$ and $y$ directions. Figure 17 shows the time

(a)

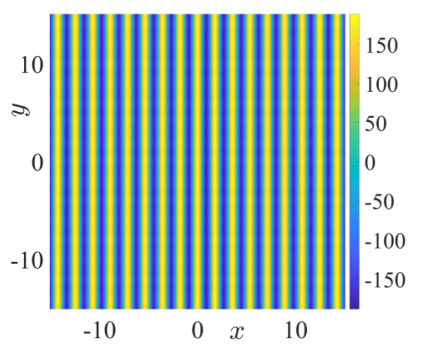

(c)

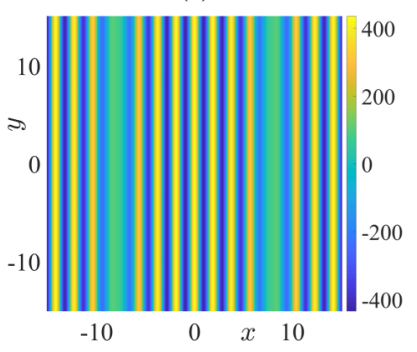

(b)

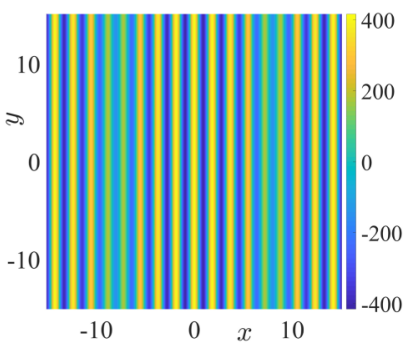

(d)

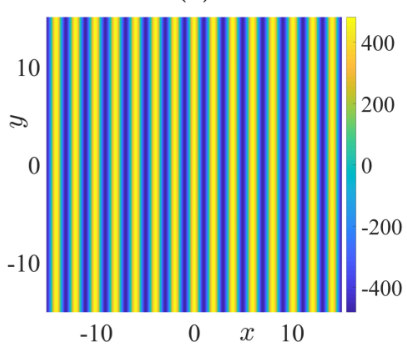

FIG. 17. Four subsequent stages in the simulation of the coupled amplitude Eqs. (51) and (52), evolving from Eckhaus unstable roll to Eckhaus stable roll. (a) $t=0$, (b) $t=27$, (c) $t=29.25$, (d) $t=33$.

evolution of the convective pattern. The system eliminates two pairs of rolls in order to augment its wavelength. After the local elimination of the wavelength, the system readjusts through a process of phase diffusion. The system reaches a wave number inside the Eckhaus stable region. Actually, the final wave number is close to $k_{c}$. Note that, unlike the one dimensional situation, where the defect exists only for an instant while a pair of rolls is created or eliminated, in two-dimensional situation, the defects persist for some time, (a)

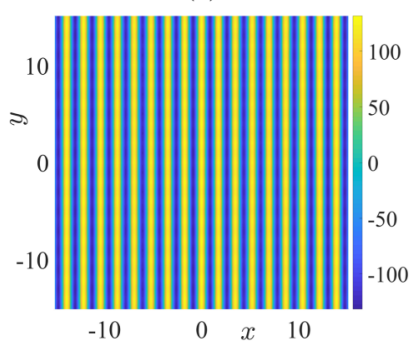

(c)

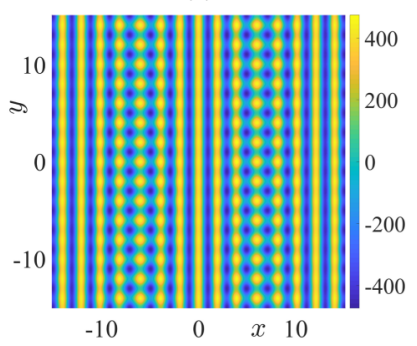

(b)

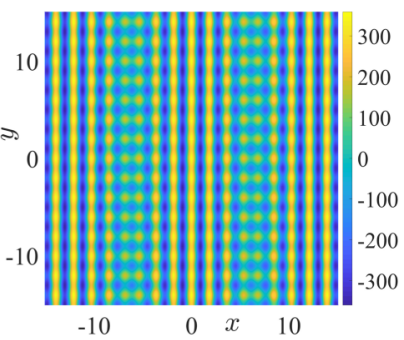

(d)

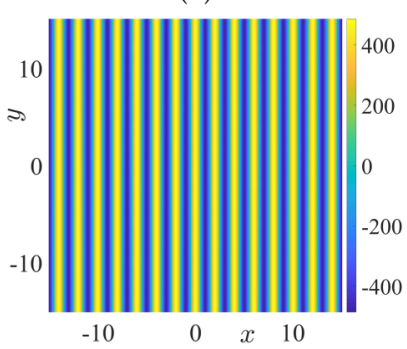

FIG. 18. Four subsequent stages in the simulation of the coupled amplitude Eqs. (51) and (52), evolving from Eckhaus and CR unstable roll to Eckhaus stable roll. (a) $t=0$, (b) $t=6$, (c) $t=8.25$, (d) $t=12$. 


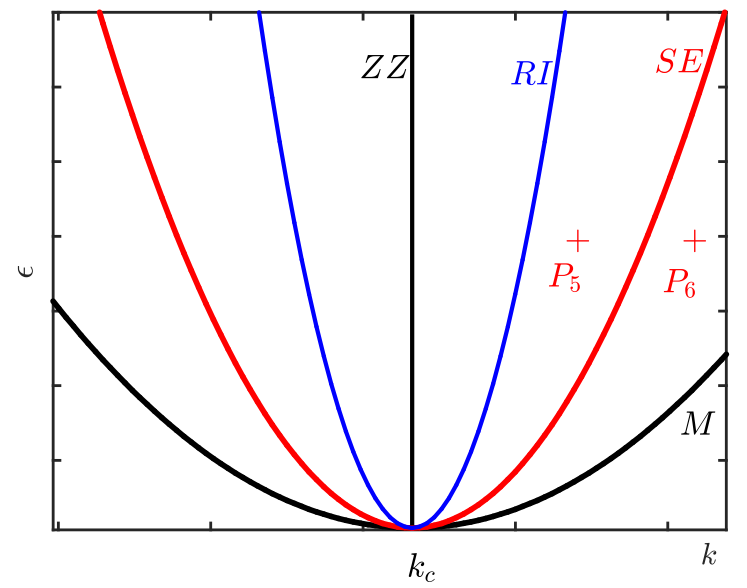

FIG. 19. Squares: Points $P_{5}, P_{6}$, where numerical simulations were performed. (M) Marginal stability curve, (SE) square Eckhaus boundary, (RI) rectangular instability boundary, (ZZ) zigzag boundary.

as shown by Figs. 17(b) and 17(c). At point $P_{4}$, the initial state is a uniform roll with $q=0.5$. In this case, the system is CR and Eckhaus unstable. Figure 18 shows the time evolution of the structure. In the first stage, a competition between CR and Eckhaus instability mechanisms is observed, before a phase diffusion process.

\section{Instability of a square solution}

Figure 19 shows two representative points, denoted by the symbol (+), where the integration of amplitude Eqs. (51) and (52) is performed.

At point $P_{5}$, we have the following parameters: $q=0.2$, $\epsilon=0.05, \alpha=5 \times 10^{-5}, \Lambda=0.01$, and $\chi=0.01$. With these (a)

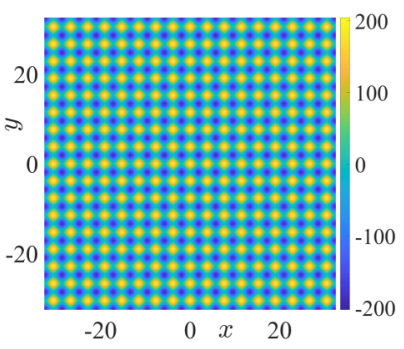

(c)

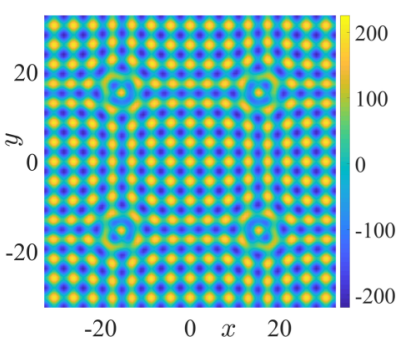

(b)

(d)

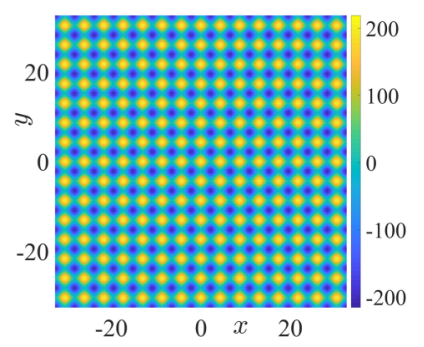

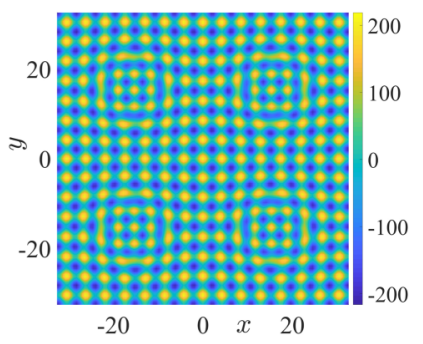

FIG. 20. Four subsequent stages in the simulation of the coupled amplitude Eqs. (51) and (52), evolving from RI unstable and SE stable square to RI stable square. (a) $t=0$, (b) $t=1900$, (c) $t=2200$, (d) $t=3000$. (a)

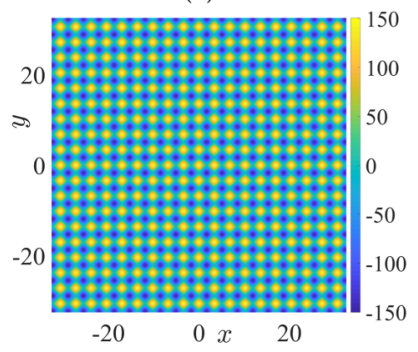

(c)

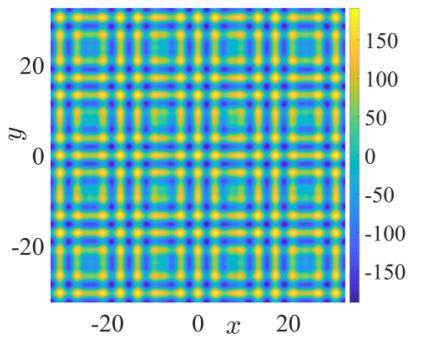

(b)

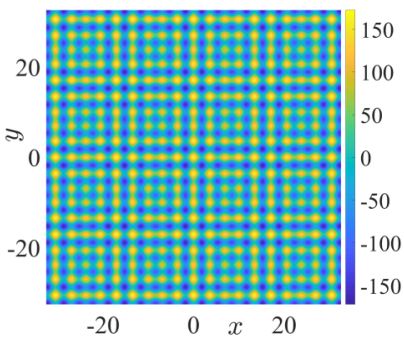

(d)

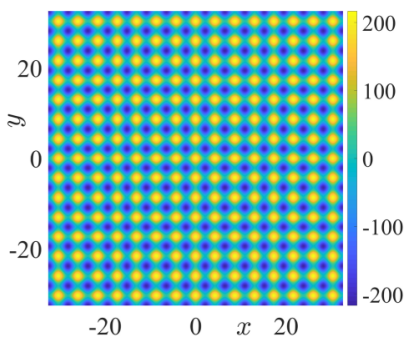

FIG. 21. Four subsequent stages in the simulation of the coupled amplitude Eqs. (51) and (52), evolving from RI and SE unstable square to RI stable square. (a) $t=0$, (b) $t=72$, (c) $t=92$, (d) $t=132$.

parameters, square solution is RI unstable and SE stable. In addition, $\left|D_{\|}\right|<D_{x y}$. Figure 20 shows the evolution of the convective pattern with time. We have represented $f(x, y, t)=$ $A(x, y, t) e^{i k_{c} x}+B(x, y, t) e^{i k_{c} y}+$ c.c. at four different chosen times. Initially, the convective pattern is a perfect square. A small random perturbation is added in the $\mathrm{x}$ and $\mathrm{y}$ directions. After elimination and adjustment of wavelengths, the structure reaches a stable state with a wave number very close to the critical value.

At point $P_{6}$, the parameters are $q=0.4, \epsilon=0.05, \alpha=$ $5 \times 10^{-5}, \Lambda=0.01$, and $\chi=0.01$. In this case, the square solution is RI and SE unstable; furthermore, $\left|D_{\|}\right|>D_{x y}$. The time evolution of the structure is shown in Fig. 21. It is not surprising that the dynamics is faster that in the previous case, since $P_{6}$ is farther for IR stability curve than $P_{5}$. The process of wavelength elimination is also quite different. This could be related to the fact that at $P_{5},\left|D_{\|}\right|<D_{x y}$, whereas at $P_{6}$, $\left|D_{\|}\right|>D_{x y}$.

\section{CONCLUSION}

In this paper, long wavelength instabilities of roll and square patterns that emerge in the Rayleigh-Bénard convection for shear-thinning fluids, in the situation where the slabs have finite conductivities and thicknesses, is studied. The influence of the shear-thinning behavior on the range of stable wave numbers and the instability mechanisms that bound the stability diagram is clearly highlighted.

The rheological behavior of fluids considered is described by the Carreau model. For this model, the rheology does not play any role on the onset of convection. The nature of the primary bifurcation and the selection of the convective pattern at threshold are investigated as a function of the shear-thinning degree $\alpha$, the slabs thickness $\Lambda$ and the ratio 
of thermal conductivities $\chi$. Comparison between the selfsaturated and the cross-saturated coefficients in the Landau equation indicates that shear-thinning effects favor formation of rolls rather than squares. Indeed, with increasing shearthinning effects, the intensity of convection increases due to a decrease of the viscosity. The nonlinearities and the coupling between the modes that constitute the square pattern become stronger, which may lead to a destabilization of the square solution. On the other hand, the intensity of convection for poorly conducting walls is lower than that for highly conducting walls, thus the critical shear-thinning degree, $\alpha_{S-R}$, below which squares are stable increases with decreasing $\chi$. The influence of the slabs thickness $\Lambda$ on $\alpha_{S-R}$ is weak when $\Lambda>1$.

The Nusselt number is roughly the same for rolls and squares and the stable structure has the highest Nusselt number, in agreement with the maximum heat transfer principle.

Subsequently, the stability of modulated rolls and squares with respect to inhomogeneous spatial perturbation is analyzed. The influence of shear-thinning effects is clearly highlighted. In the case of modulated rolls, contrary to the Newtonian case where cross-roll instability is always dominant, except at low Prandtl number, it is shown that for a non-Newtonian shear-thinning fluid, this instability prevails only when $\alpha$ is less than a critical value denoted $\alpha_{\mathrm{CR}-E}$. Under this condition, the domain of stable rolls is bounded by zigzag instability for $k<k_{c}$ and by cross-roll instability for

(a)

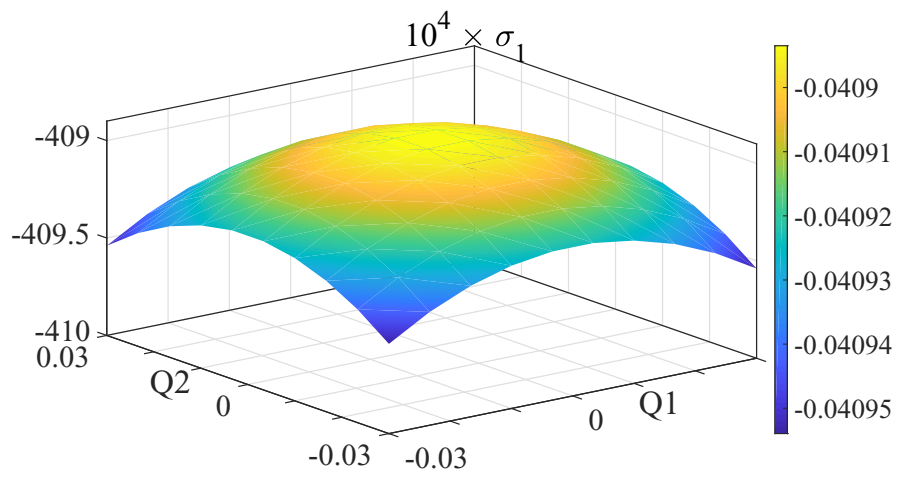

(c)

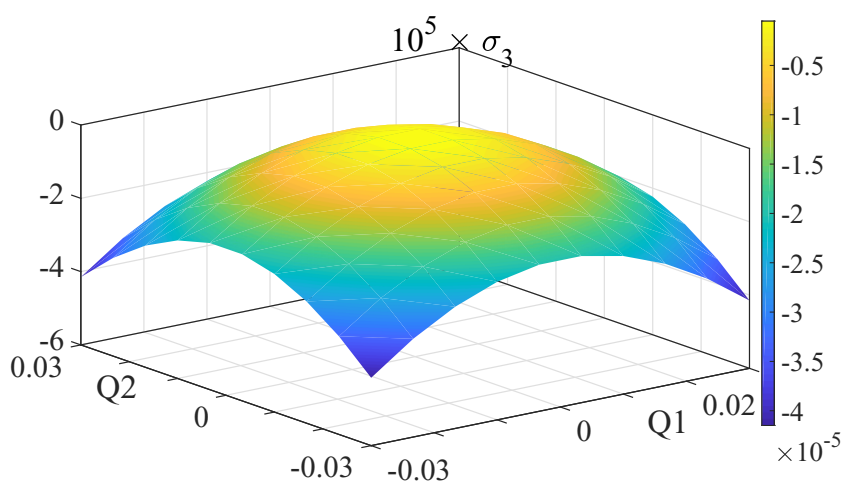

$k>k_{c}$. Furthermore, the marginal cross-roll curve enlarges with increasing shear-thinning effects. For sufficiently strong shear-thinning effects, here $\alpha>\alpha_{\mathrm{CR}-E}$, Eckhaus instability which is independent of the rheology becomes dominant. The stable rolls are bounded by zigzag and Eckhaus instabilities.

In the case of modulated squares, i.e., $\alpha<\alpha_{S-R}$, it is observed that the rectangular instability is dominant and the width of the stable wave numbers band decreases as $\alpha$ increases. The time evolution of the convective pattern initially in the unstable part of the stability-diagram is obtained from the numerical computation of the amplitude equations. The instability mechanisms are illustrated, and for all the cases considered, it is observed at the final state the structure reaches a wave number very close to the critical value.

In this study, the variation of the fluid properties and particularly the viscosity with temperature is not taken into account. Generally, non-Newtonian fluids are highly viscous and thermodependent. The thermodependency of the fluid properties leads to hexagonal patterns at the onset [45-47]. Analysis of the stability of this convective pattern is the next step of our work, dealing with the influence of the rheology on the pattern selection.

Another direction of the present study is the determination of the Busse Balloon for highly shear-thinning fluids. This is particularly interesting, since the lower part of this balloon is delimited by zigzag and Eckhaus boundaries.

FIG. 22. Variations on $Q_{1}$ and $Q_{2}$ of the four eigenvalues in the situation where the square pattern is RI unstable only. Here $\epsilon=0.1$, $q=0.3, \alpha=0.5 \times \alpha_{c}, \Lambda=1, \chi=10^{-3}$. (a) $\sigma_{1}$, (b) $\sigma_{2}$, (c) $\sigma_{3}$, (d) $\sigma_{4}$. 
(a)

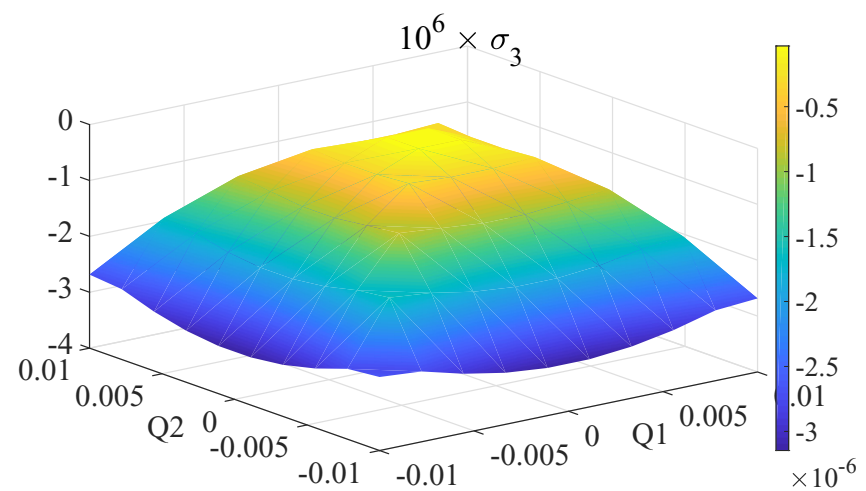

(b)

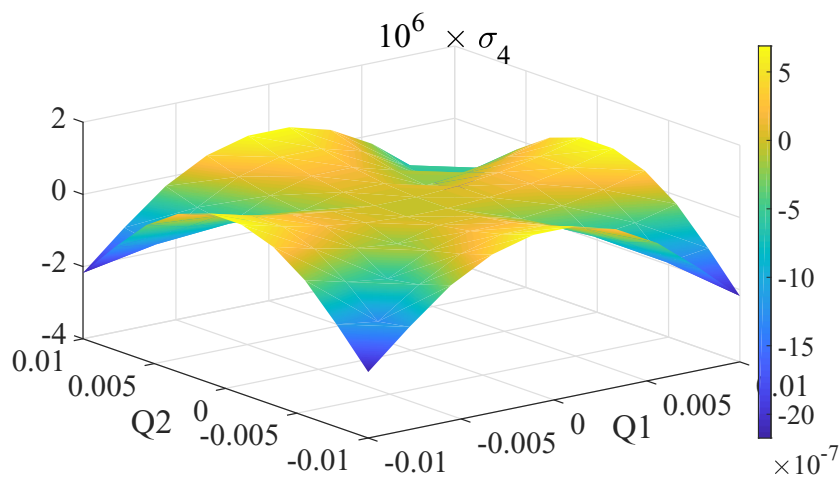

FIG. 23. Variations on $Q_{1}$ and $Q_{2}$ of the eigenvalues $\sigma_{3}$ (a) and $\sigma_{4}$ (b) in the situation where the square pattern is only zigzag unstable. Here $\epsilon=0.1, q=-0.1, \alpha=0.5 \times \alpha_{c}, \Lambda=1, \chi=10^{-3}$.

\section{APPENDIX A: EIGENVALUES VERSUS $Q_{1}$ AND $Q_{2}$ ARISING FROM THE FULL DISPERSION RELATION}

The dispersion relation arising from the system Eqs. (79)(82) is solved numerically for different values of the parameters $(q, \alpha, \epsilon, \Lambda, \chi)$. It is observed that the two first eigenvalues $\sigma_{1}$ and $\sigma_{2}$ are always negative. They are associated with the amplitudes which are damped.

Figure 22 shows the four eigenvalues as a function of $Q_{1}$ and $Q_{2}$ for the case where $\left(q+k_{c}, \epsilon\right)$ is square Eckhaus stable and Rectangular Eckhaus unstable. The eigenvalues $\sigma_{1}$ and $\sigma_{2}$ are negative as indicated above. The eigenvalue $\sigma_{3}$ is negative because the point considered is square Eckhaus stable. The eigenvalue $\sigma_{4}$ is positive and is associated with the Eckhaus rectangular instability. It is interesting to note that the maximum of $\sigma_{4}$ is reached at $\left|Q_{1}\right|=\left|Q_{2}\right|$. Further calculations indicate that for a given a wave number $q, \sigma_{4}$ increases with increasing shear-thinning effects.

The case where $\left(q+k_{c}, \epsilon\right)$, is zigzag unstable $(q<0)$ and Eckhaus stable is illustrated by Fig. 23. The eigenvalues $\sigma_{1}$ and $\sigma_{2}$ are negative and their variations on $Q_{1}$ and $Q_{2}$ are similar to those in Fig. 22 and are therefore not represented. The eigenvalue $\sigma_{3}$ is negative and may be associated with the Eckhaus stability, whereas $\sigma_{4}$ is positive and is associated with the zigzag instability which is maximum along the axis.

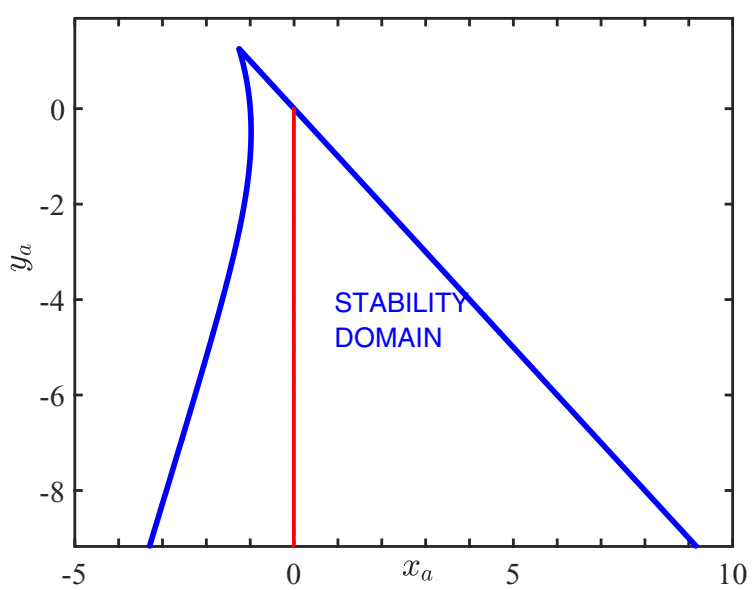

\section{APPENDIX B: STABILITY OF THE ETD2 SCHEME: EXTENSION TO TWO COUPLED GINZBURG-LANDAU EQUATIONS}

\section{Case of a single ODE}

We consider first a single ordinary differential equation (ODE) of the form

$$
\frac{d u(t)}{d t}=c u(t)+F[u(t)] .
$$

Using the second order exponential time differencing scheme for the time discretization leads to

$$
\begin{aligned}
u_{n+1}= & u_{n} e^{c \Delta t}+F_{n} \frac{(1+c \Delta t) e^{c \Delta t}-1-2 c \Delta t}{c^{2} \Delta t} \\
& +F_{n-1} \frac{1+c \Delta t-e^{c \Delta t}}{c^{2} \Delta t} .
\end{aligned}
$$

To evaluate the stability domain of this scheme, we have adopted the same approach as in Refs. [43,48]. We suppose that there is a fixed point $u_{0}$, so that $c u_{0}+F\left(u_{0}\right)=0$. Linearizing about this fixed point leads to

$$
\frac{d u(t)}{d t}=c u(t)+\lambda u(t)
$$

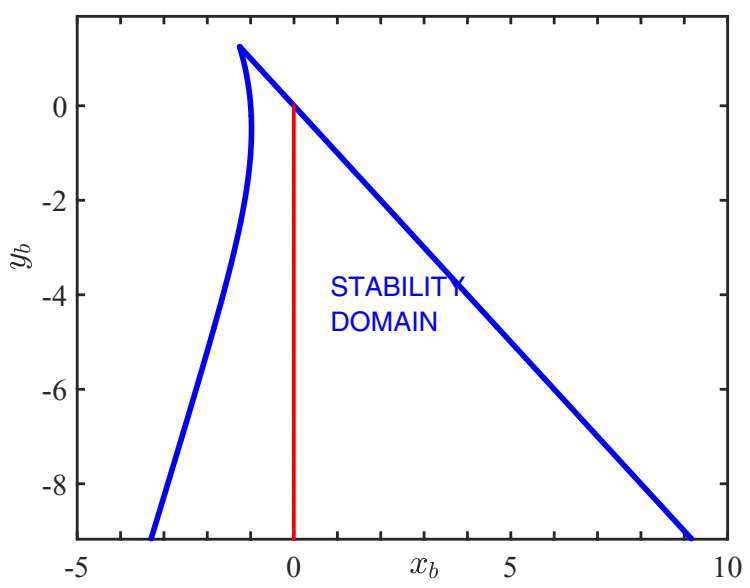

FIG. 24. The stability domain of ETD2 scheme and the repartition of the points $\left(x_{a}, y_{a}\right)$ and $\left(x_{b}, y_{b}\right)$ along the vertical line. 
where $u$ is now the perturbation to $u_{0}$ and $\lambda=F^{\prime}\left(u_{0}\right)$. The fixed point, $u_{0}$, is stable provided that $\operatorname{Re}(c+\lambda)<0$.

It can be shown straightforwardly that the fixed points of the ETD2 scheme are the same as those of the ODE (B1): consequently, the linear stability analysis of the ETD2 scheme can be performed by replacing $F_{n}$ by $\lambda u_{n}$ in Eq. (B2). A recurrence relation involving $u_{n+1}, u_{n}$ and $u_{n-1}$ is obtained:

$$
\begin{aligned}
u_{n+1}= & u_{n} e^{c \Delta t}+\lambda u_{n} \frac{(1+c \Delta t) e^{c \Delta t}-1-2 c \Delta t}{c^{2} \Delta t} \\
& +\lambda u_{n-1} \frac{1+c \Delta t-e^{c \Delta t}}{c^{2} \Delta t}
\end{aligned}
$$

Defining $r=u_{n+1} / u_{n}, \quad x=\lambda \Delta t, \quad y=c \Delta t$, the following quadratic equation for the factor $r$ by which the solution is multiplied after each step is derived:

$$
\begin{aligned}
& y^{2} r^{2}-r\left(y^{2} e^{y}+x\left[(1+y) e^{y}-2 y-1\right]\right) \\
& \quad+\left(e^{y}-1-y\right) x=0,
\end{aligned}
$$

where $r$ is the factor by which the solution is multiplied after each step so ETD2 scheme is stable provided that $|r|<1$. In general, both $c$ and $\lambda$ are complex and, consequently, the stability domain of the ETD2 scheme is four dimensional. To simplify, we choose to determine the stability region in the real plane $(\operatorname{Re}(x), \operatorname{Re}(\lambda))$. The boundaries of this domain are obtained for the values $r=1$ and $r=-1$ and correspond to the curves:

$$
y=-x \quad \text { and } \quad x=\frac{-y^{2}\left(1+e^{y}\right)}{(y+2) e^{y}-3 y-2} .
$$

\section{Extension to two coupled Ginzburg-Landau equations}

In our case, we have the following set of ODE for each couple $\left(k_{x}, k_{y}\right)$ :

$$
\begin{aligned}
& \frac{d \hat{A}_{k_{x}, k_{y}}(t)}{d t}=\left(\frac{\epsilon}{\tau_{0}}-\frac{\xi_{0}^{2}}{\tau_{0}}\left[k_{x}^{2}+\frac{k_{x} k_{y}^{2}}{k_{c}}+\frac{k_{y}^{4}}{4 k_{c}^{2}}\right]\right) \hat{A}_{k_{x}, k_{y}}(t)+\mathcal{N}_{1, k_{x} k_{y}}, \\
& \frac{d \hat{B}_{k_{x}, k_{y}}(t)}{d t}=\left(\frac{\epsilon}{\tau_{0}}-\frac{\xi_{0}^{2}}{\tau_{0}}\left[k_{y}^{2}+\frac{k_{y} k_{x}^{2}}{k_{c}}+\frac{k_{x}^{4}}{4 k_{c}^{2}}\right]\right) \hat{B}_{k_{x}, k_{y}}(t)+\mathcal{N}_{2, k_{x} k_{y}},
\end{aligned}
$$

where $\hat{A}_{k_{x}, k_{y}}(t)=\mathcal{F}(A(x, y, t))$ and $\hat{B}_{k_{x}, k_{y}}(t)=\mathcal{F}(B(x, y, t)) . \mathcal{F}($.$) designates the 2 \mathrm{D}$ discrete Fourier transform. We add small perturbations a and $\mathrm{b}$ to the initial stationary solutions $A_{0}$ and $B_{0}$. Replacing $A=A_{0}+a$ and $B=B_{0}+b$ in the former equations and after linearization, we get

$$
\begin{aligned}
\frac{d \hat{a}_{k_{x}, k_{y}}(t)}{d t}= & \left(\frac{\epsilon}{\tau_{0}}-\frac{\xi_{0}^{2}}{\tau_{0}}\left[k_{x}^{2}+\frac{k_{x} k_{y}^{2}}{k_{c}}+\frac{k_{y}^{4}}{4 k_{c}^{2}}\right]-2 g_{1}\left|A_{0}\right|^{2}-\beta\left|B_{0}\right|^{2}\right) \hat{a}_{k_{x}, k_{y}}(t) \\
& -g_{1} \mathcal{F}\left(A_{0}^{2} \bar{a}\right)_{k_{x}, k_{y}}-\beta \mathcal{F}\left(A_{0} \bar{B}_{0} b\right)_{k_{x}, k_{y}}-\beta \mathcal{F}\left(A_{0} B_{0} \bar{b}\right)_{k_{x}, k_{y}}, \\
\frac{d \hat{b}_{k_{x}, k_{y}}(t)}{d t}= & \left(\frac{\epsilon}{\tau_{0}}-\frac{\xi_{0}^{2}}{\tau_{0}}\left[k_{y}^{2}+\frac{k_{y} k_{x}^{2}}{k_{c}}+\frac{k_{x}^{4}}{4 k_{c}^{2}}\right]-2 g_{1}\left|B_{0}\right|^{2}-\beta\left|A_{0}\right|^{2}\right) \hat{b}_{k_{x}, k_{y}}(t) \\
& -g_{1} \mathcal{F}\left(B_{0}^{2} \bar{b}\right)_{k_{x}, k_{y}}-\beta \mathcal{F}\left(B_{0} \overline{A_{0}} a\right)_{k_{x}, k_{y}}-\beta \mathcal{F}\left(B_{0} A_{0} \bar{a}\right)_{k_{x}, k_{y}} .
\end{aligned}
$$

Since we consider infinitesimal perturbations, we assume that we can simplify the former equations according to

$$
\begin{aligned}
& \frac{d \hat{a}_{k_{x}, k_{y}}(t)}{d t}=\left(\frac{\epsilon}{\tau_{0}}-\frac{\xi_{0}^{2}}{\tau_{0}}\left[k_{x}^{2}+\frac{k_{x} k_{y}^{2}}{k_{c}}+\frac{k_{y}^{4}}{4 k_{c}^{2}}\right]-2 g_{1}\left|A_{0}\right|^{2}-\beta\left|B_{0}\right|^{2}\right) \hat{a}_{k_{x}, k_{y}}(t), \\
& \frac{d \hat{b}_{k_{x}, k_{y}}(t)}{d t}=\left(\frac{\epsilon}{\tau_{0}}-\frac{\xi_{0}^{2}}{\tau_{0}}\left[k_{y}^{2}+\frac{k_{y} k_{x}^{2}}{k_{c}}+\frac{k_{x}^{4}}{4 k_{c}^{2}}\right]-2 g_{1}\left|B_{0}\right|^{2}-\beta\left|A_{0}\right|^{2}\right) \hat{b}_{k_{x}, k_{y}}(t) .
\end{aligned}
$$

We deduce that the values of $c$ and $\lambda$ in this approximation are given by

$$
\begin{array}{ll}
c_{a}\left(k_{x}, k_{y}\right)=\frac{\epsilon}{\tau_{0}}-\frac{\xi_{0}^{2}}{\tau_{0}}\left[k_{x}^{2}+\frac{k_{x} k_{y}^{2}}{k_{c}}+\frac{k_{y}^{4}}{4 k_{c}^{2}}\right] ; & \lambda_{a}\left(k_{x}, k_{y}\right)=-2 g_{1}\left|A_{0}\right|^{2}-\beta\left|B_{0}\right|^{2}, \\
c_{b}\left(k_{x}, k_{y}\right)=\frac{\epsilon}{\tau_{0}}-\frac{\xi_{0}^{2}}{\tau_{0}}\left[k_{y}^{2}+\frac{k_{y} k_{x}^{2}}{k_{c}}+\frac{k_{x}^{4}}{4 k_{c}^{2}}\right] ; & \lambda_{b}\left(k_{x}, k_{y}\right)=-2 g_{1}\left|B_{0}\right|^{2}-\beta\left|A_{0}\right|^{2} .
\end{array}
$$

We have checked that for our time step $\Delta t$ and for each couple $\left(k_{x}, k_{y}\right)$, the points $\left(x_{a}, y_{a}\right)=\left(c_{a}\left(k_{x}, k_{y}\right) \Delta t, \lambda_{a}\left(k_{x}, k_{y}\right) \Delta t\right)$ and $\left(x_{b}, y_{b}\right)=\left(c_{b}\left(k_{x}, k_{y}\right) \Delta t, \lambda_{b}\left(k_{x}, k_{y}\right) \Delta t\right)$ lie in the stability domain whose boundaries are defined by Eq. (B6). To illustrate, we have represented in Fig. 24 the stability domain of the ETD2 scheme and the repartition of the points $\left(x_{a}, y_{a}\right)$ and $\left(x_{b}, y_{b}\right)$ in the case of squares for $\Lambda=0.1$ and $\chi=10^{-2}$. 
[1] A. V. Getling, Rayleigh-Bénard Convection: Structures and Dynamics, Advanced Series in Nonlinear Dynamics Vol. 11 (World Scientific, 1998).

[2] E. L. Koschmieder, Bénard Cells and Taylor Vortices (Cambridge University Press, Cambridge, 1993).

[3] E. Bodenschatz, W. Pesch, and G. Ahlers, Recent developments in Rayleigh-Bénard convection, Annu. Rev. Fluid Mech. 32, 709 (2000).

[4] N. J. Balmforth and A. C. Rust, Weakly nonlinear viscoplastic convection, J. Non-Newtonian Fluid Mech. 158, 36 (2008).

[5] B. Albaalbaki and R. Khayat, Pattern selection in the thermal convection of non-Newtonian fluids, J. Fluid Mech. 668, 500 (2011).

[6] M. Bouteraa, C. Nouar, E. Plaut, C. Metivier, and A. Kalck, Weakly nonlinear analysis of Rayleigh-Bénard convection in shear-thinning fluids: Nature of the bifurcation and pattern selection, J. Fluid Mech. 767, 696 (2015).

[7] P. Cerisier, S. Rahal, J. Cordonnier, and G. Lebon, Thermal influence of boundaries on the onset of Rayleigh-Bénard convection, Int. J. Heat Mass Transfer 41, 3309 (1998).

[8] P. Le Gal, A. Pocheau, and V. Croquette, Square Versus Roll Pattern at Convective Threshold, Phys. Rev. Lett. 54, 2501 (1985).

[9] P. Le Gal and V. Croquette, Appearance of a square pattern in a Rayleigh-Bénard experiment, Phys. Fluids 31, 3440 (1988).

[10] F. Busse and H. Riahi, Nonlinear convection in a layer with nearly insulating boundaries, J. Fluid Mech. 96, 243 (1980).

[11] M. R. E. Proctor, Planform selection by finite-amplitude thermal convection between poorly conducting slabs, J. Fluid. Mech. 113, 469 (1981).

[12] D. R. Jenkins and M. R. E. Proctor, The transition from roll to square-cell solutions in Rayleigh-Bénard convection, J. Fluid Mech. 139, 461 (1984).

[13] M. Bouteraa and C. Nouar, Weakly nonlinear analysis of Rayleigh-Bénard convection in a non-Newtonian fluid between plates of finite conductivity: Influence of shear-thinning effects, Phys. Rev. E 92, 063017 (2015).

[14] Z. Kebiche, Etude expérimentale de l'instabilité de RayleighBénard dans les fluides non-Newtoniens, Ph.D. thesis, Université de Nantes, 2014.

[15] F. Busse and J. Whitehead, Instabilities of convection rolls in a high Prandtl number fluid, J. Fluid. Mech. 47, 305 (1971).

[16] F. H. Busse and E. W. Bolton, Instabilities of convection rolls with stress-free boundaries near threshold, J. Fluid Mech. 146, 115 (1984).

[17] F. H. Busse, The oscillatory instability of convection rolls in a low Prandtl number fluid, J. Fluid Mech. 52, 97 (1972).

[18] R. B. Hoyle, Long wavelength instabilities of square patterns, Physica D: Nonlinear Phenomena 67, 198 (1993).

[19] B. Holmedal, M. Tveitereid, and E. Palm, Planform selection in Rayleigh-Bénard convection between finite slabs, J. Fluid Mech. 537, 255 (2005)

[20] A. C. Newell and J. A. Whitehead, Finite bandwidth, finite amplitude convection, J. Fluid Mech. 38, 279 (1969).

[21] P. Carriere, A. Bottaro, and P. Metzener, Wavelength selection in Rayleigh-Bénard convection between horizontal boundaries of finite conductivity, Eur. J. Mech. B, Fluids 16, 483 (1997).

[22] R. B. Bird, R. C. Armstrong, and O. Hassager, Dynamics of Polymeric Liquids: Fluids Mechanics (Wiley-Blackwell, London, 1987).
[23] Roger I. Tanner, Engineering Rheology (Oxford University Press, Oxford, 2000)

[24] D. T. J. Hurle, E. Jakeman, and E. R. Pike, On the solution of the Bénard problem with boundaries of finite conductivity, in Proceedings of the Royal Society of London A: Mathematical, Physical and Engineering Sciences (The Royal Society, London, 1967), Vol. 296, pp. 469-475.

[25] M. Rieutord, Fluid Dynamics: An Introduction (Springer, Berlin, 2014).

[26] S. Fauve, Pattern forming instabilities, in Hydrodynamics and Nonlinear Instabilities, edited by P. Huerre and M. Rossi (Cambridge University Press, Cambridge, 1998).

[27] J. T. Stuart, On the nonlinear mechanics of wave disturbances in stable and unstable parallel flows. part 1. The basic behavior in plane Poiseuille flow, J. Fluid. Mech. 9, 353 (1960).

[28] J. Watson, On the nonlinear mechanics of wave disturbances in stable and unstable parallel flows. Part 1 . The development of a solution for plane Poiseuille flow and for plane Couette flow, J. Fluid. Mech. 9, 371 (1960).

[29] T. Herbert, On perturbation methods in nonlinear stability theory, J. Fluid. Mech. 126, 167 (1983).

[30] K. Fujimura, Centre manifold reduction and the Stuart-Landau equation for fluid motions, Proc. R. Soc. London A 453, 181 (1997).

[31] M. Golubitsky, J. W. Swift, and E. Knobloch, Symmetries and pattern selection in Rayleigh-Bénard convection, Physica D: Nonlin. Phenom. 10, 249 (1984).

[32] R. Hoyle, Pattern Formation: An Introduction to Methods (Cambridge University Press, Cambridge, 2006).

[33] S. F. Liang and A. Acrivos, Experiments on buoyancy driven convection in non-Newtonian fluid, Rheol. Acta 9, 447 (1970).

[34] H. Ozoe and S. W. Churchill, Hydrodynamic stability and natural convection in Newtonian and non-Newtonian fluids heated from below, in AIChE Symposium Series, Heat Transfer, Vol. 69 (1973), pp. 126-133.

[35] W. V. R. Malkus and G. Veronis, Finite amplitude cellular convection, J. Fluid. Mech. 4, 225 (1958).

[36] A. Schlüter, D. Lortz, and F. Busse, On the stability of steady finite amplitude convection, J. Fluid Mech. 23, 129 (1965).

[37] E. Palm, A note on a maximum principle in Bénard convection, Int. J. Heat Mass Transfer 15, 2409 (1972).

[38] T. Kita, Principle of maximum entropy applied to Rayleigh-Bénard convection, J. Phys. Soc. Jpn. 75, 124005 (2006).

[39] E. M. Parmentier, A study of thermal convection in nonNewtonian fluids, J. Fluid Mech. 84, 1 (1978).

[40] L. A. Segel, Distant side walls cause slow amplitude modulation of cellular convection, J. Fluid Mech. 38, 203 (1969).

[41] M. C. Cross, Derivation of the amplitude equation at the Rayleigh-Bénard instability, Phys. Fluids 23, 1727 (1980).

[42] Y. Pomeau and P. Manneville, Stability and fluctuations of a spatially periodic convective flow, J. Phys. Lett. 40, 609 (1979).

[43] M. Steven Cox and C. Paul Matthews, Exponential time differencing for stiff systems, J. Comput. Phys. 176, 430 (2002)

[44] A. K. Kassam and L. N. Trefethen, Fourth-order time-stepping for stiff pdes, SIAM J. Sci. Comput. 26, 1214 (2005). 
[45] F. Busse, The stability of finite amplitude cellular convection and its relation to an extremum principle, J. Fluid. Mech. 30, 625 (1967).

[46] C. Q. Hoard, C. R. Robertson, and A. Acrivos, Experiments on the cellular structure in Bénard convection, Int. J. Heat Mass Transf. 13, 849 (1970).
[47] M. Darbouli, C. Métivier, S. Leclerc, C. Nouar, M. Bouteera, and D. Stemmelen, Natural convection in shear-thinning fluids: Experimental investigations by MRI, Int. J. Heat Mass Transf. 95, 742 (2016).

[48] H. Ashi, Numerical methods for stiff systems, Ph.D. thesis, University of Nottingham, 2008. 